\title{
Ethnomedicinal Study of Medicinal Plants Used by Mizo Tribes in Champhai District of Mizoram, India.
}

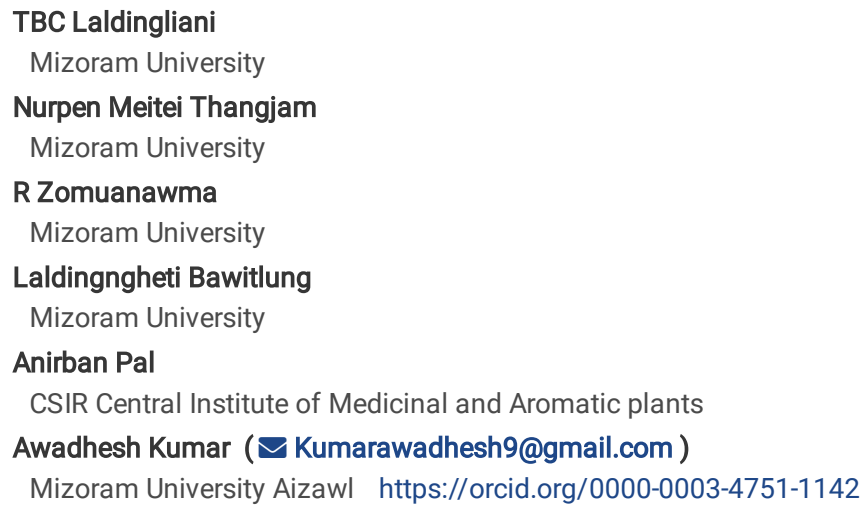

Research Article

Keywords: Ayurveda, Champhai, Ethnomedicinal, Indo-Burma Hotspot, Tribal.

Posted Date: October 29th, 2021

DOl: https://doi.org/10.21203/rs.3.rs-1021345/v1

License: (c) (1) This work is licensed under a Creative Commons Attribution 4.0 International License. Read Full License

Version of Record: A version of this preprint was published at Journal of Ethnobiology and Ethnomedicine on March 24th, 2022. See the published version at https://doi.org/10.1186/s13002-022-00520-0. 


\section{Abstract \\ Background}

Medicinal plants have been used countless times for curing diseases mainly in developing countries. They are easily available with little to no side effects when compared to modern medicine. This manuscript encompasses information on ethnomedicinal plants in Champhai district, located in the North East Region (NER) of India. The region lies within Indo-Burma biodiversity hotspot. This study will be the first quantitative report on the ethnomedicinal plants used by the local tribes of this region. Knowledge of medicinal plants is mostly acquired by word of mouth and the knowledge is dying among the local youths with the prevalence of modern medicine. Hence, there is urgency in deciphering and recording such information.

\section{Methods}

Information was gathered through interviews with 200 informants across 15 villages of the Champhai District. From the data obtained, we calculated the Used Report (UR), Frequency of Citation (FC) and Informant Consensus Factor $\left(F_{i c}\right)$ for all the plant species. Secondary data was obtained from scientific databases such as Pubmed, Sci Finder and Science Direct. The scientific name of the plants were matched and arranged in consultation with the working list of all plant species (http://www.theplantlist.org ).

\section{Results}

93 plant species from 53 families were recorded. The most common families are Euphorbiaceae and Asteraceae with six and five species representatives respectively. Leaves were the most frequently used part of a plant and were usually used in the form of decoction. The plant species with the highest used report (UR) were Curcuma longa L. (136 FC) and Flueggea virosa (126 FC). The main illness categories as per Frequency of citation were Muscle/Bone Problem $\left(0.962 \mathrm{~F}_{\mathrm{ic}}\right)$, Gastro-intestinal Disease $\left(0.956 \mathrm{~F}_{\mathrm{ic}}\right)$ and Skin Care $\left(0.953 \mathrm{~F}_{\mathrm{ic}}\right)$.

\section{Conclusion}

The people of Mizoram living in the Champhai district have an immense knowledge of ethnomedicinal plants. There are no new adverse effects recorded. We observed that there is a scope of scientific validation of 10 plant species for their pharmacological activity and 13 species for the phytochemical characterization or isolation of the phytochemicals. This might pave the path for developing a scientifically validated botanical or lead to semisyntheic derivatives intended for modern medicine.

\section{Background}

Plants have been known to be a major source of diverse chemical compounds possessing both medicinal properties and commercial value. There have been several reports on medicinal plants as a source for drug discovery. However, new diseases will likely continue to emerge along with drug-resistant pathogens. This dynamic nature of pathogens has constantly challenged researchers to look for alternatives. The past few decades have witnessed the surge in ethnomedicinal plant research [1], one of the reasons being that the natural products have played an important role in the development of drugs, contributing more than $50 \%$ of clinical drugs in the pharmaceutical industry [2]. Further, the rapid growth in human population has raised the demand which inturn has increased the quest for novel plant resources, triggering a threat to natural resources [3].

Traditional knowledge and practices of herbal remedies have been passed on to new generations over the centuries and will continue to do so, with some variations taking place every generation. From the 19th century till date, plants have become the main source for therapeutic regimes and traditional practices are proven to have little known side effects besides their low cost and easy availability. India has been well known worldwide for its indigenous traditional pieces of knowledge and practices from ancient times, through different systems of medicine such as Ayurveda, Siddha and Unani [4]. Although more than 427 tribal communities are having vast diversity of ancient traditions, still there has been criticism of ethnomedicines due to regional variation, political and socio-economic challenges [5]. Reports are stating that several plants have been increasingly utilised by the indigenous people of India [1]. Generally, in India, it was estimated that 6,000 flora are used in traditional and herbal medicine which represent about $75 \%$ of the needs of the third world and meanwhile 3,000 plants were officially acknowledged due to their medicinal values [6].

The healthcare system of India witnesses a wide variation encompassing urban and rural populations which rely on both modern and traditional systems of medicine. The recently implemented Ayushman Bharat Pradhan Mantri Jan Arogya Yojana from the Commonwealth Fund enables cashless secondary and tertiary care at private facilities. [7]. besides, health insurance schemes also exist for institutions and factories. Catering to the huge population has its limitations and thus many of the ailments are treated either by traditional healers or through traditional knowledge and practices especially in remote areas. One such state in the North-Eastern part of India is Mizoram.

The ethnomedicinal usages [8-11] and the flora $[12,13]$ in the state of Mizoram though has been previously reported, however, there is still a scope considering the ethnomedicinal knowledge of the diverse tribes within the state. Hence, through the present manuscript, we aim to document the ethnomedicinal practices involving medicinal plants of the Champhai district of Mizoram, India. Their practical knowledge has been established based on more than a century of credence and observation. 
Mizoram lies within the Indo-Burma biodiversity hotspot region and shares two international borders with Bangladesh in the west and Myanmar in the east. According to Champion and Seth (1968), Mizoram forests are classified into Tropical semi-evergreen forests, tropical wet evergreen forests and mountain subtropical pine forests [14]. The study area, i.e., the Champhai district is classified as a rural area where health care facilities are relatively poor which drives the people to rely on traditional medicines. The traditional healers using medicinal plant-based formulations for various ailments indicate that traditional medicines are still one of the mainstays in their contemporary health care. It is felt that prospection and research on the medicinal plants that play such an important role in the health care of Mizo tribes need a more intensified effort.

\section{Methods \\ Description of study Area}

Champhai is one of the 8 districts in Mizoram, amidst the North East Region of India. It is located in the eastern part of Mizoram, internationally bordered by Myanmar and therefore becoming the main gate of trading for India and Myanmar. It lies between $23.456^{\circ} \mathrm{N}$ latitude and $93.328^{\circ} \mathrm{E}$ longitude. The average annual rainfall is approximately $1814 \mathrm{~mm}$ and the temperature remains around $18.6^{\circ} \mathrm{C}$ which is slightly colder than the rest of the state during winter. The total land area is $3185.83 \mathrm{sq}$ kilometres at an elevation around 1,678 $\mathrm{m}$ above sea level, population density is 10 per sq kilometres (32,734). According to an official Census (2011), Champhai reported a population of 1.26 lakhs, of which male and female were 62,357 and 63,388 respectively [15, 16]. The study area was divided into 15 village council areas (Vengthlang, Vengthlang North, Venglai, Vengsang, Electric veng, Kanan, Kahrawt, Bethel, New Champhai, Zotlang, Hmunhmeltha, Tualcheng, Ngopa, Khawzawl and East Lungdar) for extensive data collection (Fig. 1). The majority of people living in this area are Mizo tribe and use the Mizo dialect in common.

\section{Investigative method}

In the field study, formal questionnaires were distributed to each participant while having face to face interviews at their residence. At least 16 people were interviewed in each village council area. Only those people who were skilled in the art of preparing medicines either for their families or their neighbourhood were considered for the interaction. The interactions primarily focussed on their experience, type of dosage form, duration of usage, any adverse effects observed and the source of their knowledge about the plant and their parts used. This information was then correlated with the scientific data curated from related databases (Pubmed, SciFinder and Science Direct). In most of the cases, the voucher specimens were deposited (Herbarium, Mizoram University, Aizawl, Mizoram, India) for their authentication and archiving.

\section{Characteristics of demographic data}

This demonstrated the socio-economic information of the informant including qualities like age, sex, education level and occupation. 200 persons between the ages group of 18-71 years were interviewed, of which 112 and 82 were males and females respectively. Respondents belonged to various professions while some were students. The feature of demographical characteristics obtained in the study is tabulated below (Table 1).

\section{Quantitative analysis}

\section{Frequency of citation (FC)}

Frequency of citation was used to further examine the primary data by finding the sum of total citations/usage reports for a particular species. The usage report is the quotation of one plant by an informant [17].

\section{Use Value}

Use Value or UV is used to express the correlative importance of each particular plant species locally known and was calculated by the following equation [18].

$$
U V=\sum \frac{U_{i}}{n}
$$

Where ' $U_{i}$ ' represents the number of citations of each species by the informants and ' $n$ ' represents the total number of informants in the study area. The larger the number of citations, the greater is the use-value.

\section{Informant Consensus Factor}

$F_{\text {ic }}$ or ICF is used to represent the consistency of the information among the informants, indicating whether there was shared knowledge and concurrence in the use of plants for treating the ailment category amongst the plant's users in the study area. It was calculated by the following equation [19].

$$
F_{i c}=\frac{\mathrm{N}_{u r}-N_{t}}{\mathrm{~N}_{u r}-1}
$$

Where ' $\mathrm{N}_{\mathrm{ur}}$ ' refers to the number of users reports in each illness category and ' $\mathrm{N}_{\mathrm{t}}$ ' refers to the number of plant species used for a particular illness category by all the informants.

\section{Results}

\section{Demographic characteristics}


All the 200 respondents were randomly selected from 15 village council areas interviewing at least 16 persons in each area with no equal separation of malefemale ratio. Amongst them, the elderly in their seventies and above occupied $6.5 \%$ only, while people between $31-50$ years old occupied $34.5 \%$. The average age among the informants was 54 years. Mizoram is the second most literate state in the country ounds(2011 census) and all the informants were literate having at least primary school level education. Out of the total informants, $32.5 \%$ were engaged in government jobs like teachers, officers, while $35 \%$ were selfemployed like farmers, carpenters, skilled workers, small businesses and the rest $32.5 \%$ of the informants were unemployed including students and housewives (Table 1).

Table 1

Demographic characteristics of informants $(n=200)$

\begin{tabular}{|lll|}
\hline Demographic characteristics & & \\
\hline Age & Number & In percent (\%) \\
\hline $18-30$ & 42 & 21 \\
\hline $31-50$ & 69 & 34.5 \\
\hline $51-70$ & 76 & 38 \\
\hline 71 above & 13 & 6.5 \\
\hline Sex & & \\
\hline Male & 112 & 56 \\
\hline Female & 88 & 44 \\
\hline Educational level & & \\
\hline Primary & 30 & 15 \\
\hline Middle & 44 & 22 \\
\hline High School & 56 & 28 \\
\hline Higher Secondary & 32 & 16 \\
\hline University & 38 & 19 \\
\hline Occupation & 65 & 32.5 \\
\hline $\begin{array}{l}\text { Self Employed } \\
\text { (farmer, carpenter, bussiness) }\end{array}$ & 70 & \\
\hline $\begin{array}{l}\text { Govt. Employed } \\
\text { (teacher, bank, officer) }\end{array}$ & & \\
\hline Unemployed & 65 & \\
Student, housewives) & & \\
\hline & & \\
\hline
\end{tabular}

\section{Taxonomy Identification}

In the present study, 93 medicinal plant species belonging to 53 families have been reported for treating various kinds of ailments. The most prominent families were Euphorbiaceae with 6 plant species followed by Asteraceae with 5 plant species and 4 species each among Cucurbitaceae and Zingiberaceae. Liliaceae, Fabaceae, Verbenaceae, Solanaceae, Rutaceae, Anacardiaceae are with 3 species each while Orchidaceae, Combrethaceae Theaceae, Arecaceae, Apocynaceae, Musaceae, Rubiaceae, Schrophulariaceae, Lamiaceace, Mimosaceae, Smilacaceae are with 2 species each and other 34 families with one species each as shown in Table 2. The high usage report of this large family like Euphorbiaceae (6 species), Asteraceae (5 species) and Zingiberaceae (4 species) occupied $10.8 \%, 9.2 \%$ and $8.35 \%$ of the total used report respectively indicating that most people in the study area are inclined to use plants that are easily available and abundant around them (Table 2). 
Table 2

Family with their used report and in percent

\begin{tabular}{|c|c|c|c|}
\hline Family & $\begin{array}{l}\text { No of } \\
\text { species }\end{array}$ & No of used report & $\%$ \\
\hline Liliaceae & 3 & 76 & 2.8 \\
\hline Apocynaceae & 2 & 53 & 1.95 \\
\hline Bromoliaceae & 1 & 49 & 1.8 \\
\hline Ochidaceae & 2 & 14 & 0.52 \\
\hline Combretaceae & 2 & 55 & 1.66 \\
\hline Euphorbiaceae & 6 & 294 & 10.8 \\
\hline Meliaceae & 2 & 54 & 1.99 \\
\hline Begoniaceae & 1 & 37 & 1.36 \\
\hline Cucurbitaceae & 4 & 82 & 3.02 \\
\hline Amarathaceae & 1 & 15 & 0.55 \\
\hline Betuliaceae & 1 & 7 & 0.26 \\
\hline Fabaceae & 3 & 56 & 2.06 \\
\hline Verbenaceae & 3 & 78 & 2.87 \\
\hline Theaceae & 2 & 28 & 1.03 \\
\hline Cannabinaceae & 1 & 36 & 1.32 \\
\hline Apiaceae & 1 & 29 & 1.07 \\
\hline Solanaceae & 3 & 83 & 3.05 \\
\hline Caricaceae & 1 & 53 & 1.95 \\
\hline Fagaceae & 1 & 17 & 0.63 \\
\hline Zingiberaceae & 4 & 227 & 8.35 \\
\hline Asteraceae & 5 & 250 & 9.2 \\
\hline Rutaceae & 3 & 120 & 4.42 \\
\hline Araceae & 1 & 2 & 0.07 \\
\hline Dilleniaceae & 1 & 22 & 0.81 \\
\hline Discoreaceae & 1 & 27 & 0.99 \\
\hline Caryophyllaceae & 1 & 28 & 1.03 \\
\hline Eleagnaceae & 1 & 31 & 1.14 \\
\hline Areaceae & 2 & 86 & 3.17 \\
\hline Musaceae & 3 & 80 & 2.94 \\
\hline Rubiaceae & 2 & 38 & 1.4 \\
\hline Protiaceae & 1 & 15 & 0.55 \\
\hline Malvaceae & 1 & 4 & 0.15 \\
\hline Saurauraceae & 1 & 16 & 0.59 \\
\hline Convulvulaceae & 1 & 37 & 1.36 \\
\hline Rosaceae & 1 & 14 & 0.52 \\
\hline Schrophulariaceae & 2 & 48 & 1.77 \\
\hline Campanulaceae & 1 & 25 & 0.92 \\
\hline Myrsinaceae & 1 & 4 & 0.15 \\
\hline Magnoliaceae & 1 & 18 & 0.66 \\
\hline Anacardiaceae & 3 & 79 & 2.91 \\
\hline
\end{tabular}

Page 5/30 


\begin{tabular}{|llll|}
\hline Family & $\begin{array}{l}\text { No of } \\
\text { species }\end{array}$ & No of used report & $\%$ \\
\hline Lamiaceae & 2 & 40 & 1.47 \\
\hline Clusiaceae & 1 & 18 & 0.66 \\
\hline Mimosaceae & 2 & 53 & 1.95 \\
\hline Moraceae & 1 & 2 & 0.07 \\
\hline Bignoliaceae & 1 & 37 & 1.36 \\
\hline Pandanaceae & 1 & 24 & 0.88 \\
\hline Phyllanthaceae & 1 & 48 & 1.77 \\
\hline Plantaginaceae & 1 & 36 & 1.32 \\
\hline Myrtaceae & 1 & 98 & 3.61 \\
\hline Polypodiaceae & 1 & 12 & 0.44 \\
\hline Punicaceae & 1 & 31 & 1.14 \\
\hline Smilacaceae & 2 & 26 & 0.96 \\
\hline Poaceae & 1 & 15 & 0.55 \\
\hline
\end{tabular}

\section{Frequency of usage of parts of plants}

The most commonly used medicinal plants fell under herbs (35.5\%) followed by trees (33.3\%), shurbs (18.3\%) and creepers (12.9\%) as shown in (Fig. 3.). Amongst the parts, leaves, fruits and barks were mainly utilised by the informants. (Fig. 4.) A detailed analysis concluded that leaves (47\%) followed by fruits $(14 \%)$, barks $(11 \%)$, seeds $(10 \%)$, rhizomes $(6 \%)$, stems $(4 \%)$, young shoot $(2 \%)$, oil $(1 \%)$ and in some cases the whole plant $(3 \%)$ were used for ethnomedicinal purposes.

\section{Mode of preparation and administration}

The mode of formulation preparation or administration was observed to be in the form of decoction (44.2\%) followed by paste (23\%), raw (19.5\%), juice (9.73\%), powder (1.77\%) and others like maceration and oil (1.77\%) (Fig. 5.). 
Table 3

List of plant species with their Use Value (UV)

\begin{tabular}{|c|c|c|c|c|c|c|c|c|c|c|}
\hline $\begin{array}{l}\text { Sl. } \\
\text { No. }\end{array}$ & $\begin{array}{l}\text { Species Name; } \\
\text { Voucher No }\end{array}$ & Family Name & Local Name & ${ }^{\text {a Habit }}$ & $\begin{array}{l}\text { bPart } \\
\text { Use }\end{array}$ & $\begin{array}{l}\text { Mode of } \\
\text { administration }\end{array}$ & Ailments \& UR & FC & UV & $\begin{array}{l}\text { Isolated Chemic: } \\
\text { Compounds }\end{array}$ \\
\hline 1 & $\begin{array}{l}\text { Allium cepa } \\
\text { Linn. } \\
\text { (HAMP20045) }\end{array}$ & Liliaceae & Purun sen & $\mathrm{H}$ & $\begin{array}{l}\text { Rt } \\
\text { (Bulb) }\end{array}$ & Raw & $\begin{array}{l}\text { Hair care (12), } \\
\text { headache (1) }\end{array}$ & 13 & 0.065 & $\begin{array}{l}\text { S-alk(en)yl-subsi } \\
\text { cysteine sulphox } \\
\text { quercetin, kaemf }\end{array}$ \\
\hline 2. & $\begin{array}{l}\text { Allium sativum } \\
\text { L. (HAMP20046) }\end{array}$ & Liliaceae & Purun var & $\mathrm{H}$ & $\begin{array}{l}\text { Rt } \\
\text { (Bulb) }\end{array}$ & Raw & $\begin{array}{l}\text { Tension } \\
(10), \\
\text { toothache (2), } \\
\text { cold (12), } \\
\text { pimple (7) }\end{array}$ & 31 & 0.155 & $\begin{array}{l}\text { S-allyl-cysteine, } \\
\text { thiosulfinate (All } \\
\text { ally-mercapto cy } \\
25]\end{array}$ \\
\hline 3 & $\begin{array}{l}\text { Aloe vera (L.) } \\
\text { Burm.f. } \\
\text { (HAMP20047) }\end{array}$ & Liliaceae & Liliaceae & $\mathrm{H}$ & Lf & Raw & $\begin{array}{l}\text { Burn (21), } \\
\text { Stomachache } \\
\text { (2), skin care } \\
\text { (9) }\end{array}$ & 32 & 0.16 & $\begin{array}{l}\text { Vitamin E, Sulful } \\
\text { butyl heptadecyl } \\
\text { Tetradecyne [29] }\end{array}$ \\
\hline 4 & $\begin{array}{l}\text { Alstonia } \\
\text { scholaris (L.) R. } \\
\text { Br. } \\
\text { (HAMP20006) }\end{array}$ & Apocynaceae & Thuamriat & $\mathrm{T}$ & $\mathrm{Br}$ & Decoction & Ulcer (15) & 15 & 0.075 & $\begin{array}{l}\text { Alstonine, picrini } \\
\text { akuammicine, ec } \\
\text { [33] }\end{array}$ \\
\hline 5 & $\begin{array}{l}\text { Ananas } \\
\text { comosus (L.) } \\
\text { Merr. } \\
\text { (HAMP20019) }\end{array}$ & Bromeliaceae & Lakhuihthei & Sh & $\mathrm{Lf}, \mathrm{Fr}$ & Raw, paste & $\begin{array}{l}\text { Ulcer (12), } \\
\text { seizure (1), } \\
\text { Hypertension } \\
\text { (10), urinary } \\
\text { infection (16), } \\
\text { lung disease } \\
\text { (10) }\end{array}$ & 49 & 0.245 & $\begin{array}{l}\text { 1-0-feruloylglyce } \\
\text { 2, 4-dichloroben: } \\
\text { etc [36] }\end{array}$ \\
\hline 6 & $\begin{array}{l}\text { Anoectochilus } \\
\text { brevilabris Lindl. } \\
\text { (HAMP20062) }\end{array}$ & Orchidaceae & Hnah mawi & $\mathrm{H}$ & Lf & Paste & $\begin{array}{l}\text { Pile problem } \\
\text { (12) }\end{array}$ & 12 & 0.06 & Not reported \\
\hline 7 & $\begin{array}{l}\text { Anogeissus } \\
\text { acuminata } \\
\text { (Roxb. ex DC.) } \\
\text { Wall. Ex Guillem. } \\
\text { \&Perr. } \\
\text { (HAMP20023) }\end{array}$ & Combretaceae & Zairum & $\mathrm{T}$ & $\mathrm{Br}$ & Decoction, & Ulcer (26) & 26 & 0.13 & $\begin{array}{l}\text { Castamollilin, } \mathrm{Gr} \\
\text { (-)-Secoisolaricir } \\
\text { bis-(4-Hydroxyb } \\
\text { butadiene [39] }\end{array}$ \\
\hline 8 & $\begin{array}{l}\text { Aporosa } \\
\text { octandra (Buch- } \\
\text { Ham. ex D. Don) } \\
\text { Vickery } \\
\text { (HAMP20035) }\end{array}$ & Euphorbiaceae & Chhawntual & $\mathrm{T}$ & $\mathrm{Lf}, \mathrm{Br}$ & Decoction & $\begin{array}{l}\text { Ulcer (5), } \\
\text { uterus problem } \\
(11)\end{array}$ & 16 & 0.08 & $\begin{array}{l}\text { 2-Methyl-3-en-bu } \\
\text { cyclohexyl Phth' } \\
\text { Coclaurine (AO-5 }\end{array}$ \\
\hline 9 & $\begin{array}{l}\text { Azadirachta } \\
\text { indica A. Juss } \\
\text { (HAMP20052) }\end{array}$ & Meliaceae & Neem & $\mathrm{T}$ & Lf & Decoction & $\begin{array}{l}\text { Malaria (16), } \\
\text { jaundice (8) }\end{array}$ & 24 & 0.12 & $\begin{array}{l}\text { Diepoxyazadirol, } \\
\text { flowerone, O-me1 } \\
\text { onolide [46] }\end{array}$ \\
\hline
\end{tabular}

\begin{tabular}{|c|c|c|c|c|c|c|c|c|c|c|}
\hline 10 & $\begin{array}{l}\text { Begonia inflata } \\
\text { C.B. Clark. } \\
\text { (HAMP20016) }\end{array}$ & Begoniaceae & Sekhupthur & $\mathrm{H}, \mathrm{Cr}$ & Lf & Paste, juice & $\begin{array}{l}\text { Pile problem } \\
(12), \text { diarrhea } \\
(11), \text { dysentery } \\
(14)\end{array}$ & 37 & 0.185 & Not reported \\
\hline 11 & $\begin{array}{l}\text { Benincasa } \\
\text { hispida (Thunb.) } \\
\text { Cogn. } \\
\text { (HAMP20028) }\end{array}$ & Cucurbitaceae & Maipawl & $\mathrm{H}, \mathrm{Cr}$ & $\mathrm{Fr}$ & Decoction & $\begin{array}{l}\text { Stomach } \\
\text { problem (4), } \\
\text { digestion (10), } \\
\text { diarrhoea (15) }\end{array}$ & 29 & 0.145 & $\begin{array}{l}\text { Pentanoic acid, ! } \\
\text { 2,4-dibutyl phen } \\
\text { Palmitic acid, 9,1 } \\
\text { Octadeca-dienylı } \\
\text { [48] }\end{array}$ \\
\hline
\end{tabular}

\begin{tabular}{|c|c|c|c|c|c|c|c|c|c|c|}
\hline 12 & $\begin{array}{l}\text { Beta vulgaris L. } \\
\text { (HAMP20001) }\end{array}$ & Amaranthaceae & Beet root & $\mathrm{H}$ & $\mathrm{Rh}$ & Raw, juice & $\begin{array}{l}\text { Aneamia (3), } \\
\text { immuno- } \\
\text { booster (8), } \\
\text { cancer (4) }\end{array}$ & 15 & 0.075 & $\begin{array}{l}\text { Apigenin, Luteoli } \\
\text { Isoscutellarein } 7 \\
\text { glucosyl, 8-0-xyl } \\
\text { Caffeoyl-6-(3,4-d } \\
\text { benzoyl) } \beta \text {-D-glu }\end{array}$ \\
\hline
\end{tabular}

Habit: H-Herbs; Sh-Shurbs; Cr-Creeper; T-Tree UR-Used reports FC-Frequency of citation UV-Use value

bPart used: Lf-Leaf; Br-Bark; Fr-Fruit, Rh-Rhizome; St-Stem; S-Seed; WP-Whole Plants; YS-Young Shoot 


\begin{tabular}{|c|c|c|c|c|c|c|c|c|c|c|}
\hline $\begin{array}{l}\text { Sl. } \\
\text { No. }\end{array}$ & $\begin{array}{l}\text { Species Name; } \\
\text { Voucher No }\end{array}$ & Family Name & Local Name & ${ }^{\text {a Habit }}$ & $\begin{array}{l}\text { bPart } \\
\text { Use }\end{array}$ & $\begin{array}{l}\text { Mode of } \\
\text { administration }\end{array}$ & Ailments \& UR & FC & UV & $\begin{array}{l}\text { Isolated Chemic } \\
\text { Compounds }\end{array}$ \\
\hline 13 & $\begin{array}{l}\text { Betula alnoides } \\
\text { Buch-Ham. ex D. } \\
\text { Don } \\
\text { (HAMP20017) }\end{array}$ & Betulaceae & Hriang & $\mathrm{T}$ & Lf & Paste & $\begin{array}{l}\text { Tooth paste } \\
\text { (7) }\end{array}$ & 7 & 0.035 & $\begin{array}{l}\text { a-pinene; a-terpir } \\
\text { limonene; campl } \\
\text { pinene [55] }\end{array}$ \\
\hline 14 & $\begin{array}{l}\text { Bischofia } \\
\text { javanica Blume } \\
\text { (HAMP20036) }\end{array}$ & Euphorbiaceae & Khuang thli & $\mathrm{T}$ & Lf & Paste & $\begin{array}{l}\text { Toothache } \\
\text { (13) }\end{array}$ & 13 & 0.065 & $\begin{array}{l}\text { 3,4-dihydroxyph } \\
\text { alcohol, isotachi } \\
\text { catechin, epicate } \\
\text { gallocatechin [5: }\end{array}$ \\
\hline 15 & $\begin{array}{l}\text { Cajanus cajan } \\
\text { (L.) Millsp. } \\
\text { (HAMP20041) }\end{array}$ & Fabaceae & Behliang & Sh & Lf & Decoction & $\begin{array}{l}\text { Jaundice (25), } \\
\text { intestinal } \\
\text { worms (3) }\end{array}$ & 28 & 0.14 & $\begin{array}{l}\text { 3,5-bis1,1-dimetr } \\
\text { Tetradecanoic ar } \\
\text { hexadecyl, ester, } \\
\text { Hexadecanoic ai }\end{array}$ \\
\hline 16 & $\begin{array}{l}\text { Callicarpa } \\
\text { arborea Roxb. } \\
\text { (HAMP20087) }\end{array}$ & Verbenaceae & Hnah kiah & $\mathrm{T}$ & $\mathrm{Lf}, \mathrm{Br}$ & Decoction & $\begin{array}{l}\text { Stomach ache } \\
\text { (9), diabetes } \\
\text { (16), } \\
\text { convulsion (7) }\end{array}$ & 32 & 0.16 & $\begin{array}{l}\text { Martynoside, } \\
\text { Isomartynoside, } \\
\text { acid, Antiarol rut } \\
\text { [62] }\end{array}$ \\
\hline 17 & $\begin{array}{l}\text { Camellia } \\
\text { sinensis (L.) } \\
\text { Kuntze } \\
\text { (HAMP20082) }\end{array}$ & Theaceae & Thingpuife & Sh & Lf & $\begin{array}{l}\text { Raw, } \\
\text { Decoction }\end{array}$ & $\begin{array}{l}\text { Toothace (7), } \\
\text { itchy eye (7) }\end{array}$ & 14 & 0.07 & $\begin{array}{l}\text { Caffeine, Theobr } \\
\text { Gallic acid, Amp } \\
\text { epicatechin-3-0- } \\
\text { catechin-3-0-gal }\end{array}$ \\
\hline 18 & $\begin{array}{l}\text { Cannabis sativa } \\
\text { L. (HAMP20021) }\end{array}$ & Cannabinacae & Trip/ Kanza & $\mathrm{H}$ & Lf & Raw, & $\begin{array}{l}\text { Stomach ache } \\
\text { (12), diarrhea } \\
(24)\end{array}$ & 36 & 0.18 & $\begin{array}{l}\text { Cannabigerol, } \\
\text { cannabichromer } \\
\text { cannabidiol, can } \\
\text { delta 9- } \\
\text { Tetrahydrocanna }\end{array}$ \\
\hline 19 & $\begin{array}{l}\text { Centella asiatica } \\
\text { (L.) Urb. } \\
\text { (HAMP20005) }\end{array}$ & Apiaceae & $\begin{array}{l}\text { Darbengbur } \\
\text { /lambak }\end{array}$ & $\mathrm{H}$ & WP & Decoction & $\begin{array}{l}\text { Stomach ache } \\
\text { (8), urinary } \\
\text { infection (17), } \\
\text { kidney disease } \\
\text { (2), eye pain } \\
\text { (2) }\end{array}$ & 29 & 0.145 & $\begin{array}{l}\text { Centellin, asiatic } \\
\text { centellicin [68] }\end{array}$ \\
\hline 20 & $\begin{array}{l}\text { Capsicum } \\
\text { annuum L. } \\
\text { (HAMP20084) }\end{array}$ & Solanaceae & Hmarhcha & $\mathrm{H}$ & $\mathrm{Fr}$ & Paste & $\begin{array}{l}\text { Wasp sting (9), } \\
\text { burn (3), } \\
\text { toothache (4), } \\
\text { boil (2) }\end{array}$ & 18 & 0.09 & $\begin{array}{l}\text { Ascorbic acids, c } \\
\text { luteolin, chrysoe } \\
\text { hydroxycinnami }\end{array}$ \\
\hline 21 & $\begin{array}{l}\text { Carica papaya L. } \\
\text { (HAMP20026) }\end{array}$ & Caricaceae & Thingfanghma & $\mathrm{T}$ & $\begin{array}{l}\text { Lf, Fr } \\
\text { sap }\end{array}$ & Paste, raw & $\begin{array}{l}\text { Tonsil (1), face } \\
\text { pack (3), } \\
\text { cancer (16), } \\
\text { diabetes (11), } \\
\text { dog bite (17), } \\
\text { milk booster in } \\
\text { mother (5) }\end{array}$ & 53 & 0.265 & $\begin{array}{l}\text { Benzyl- } \beta \text {-d gluco } \\
\text { sistosterol, } \beta \text {-sis } 1 \\
\text { oleic acids [74] }\end{array}$ \\
\hline 22 & $\begin{array}{l}\text { Castanopsis } \\
\text { tribuloides (Sm.) } \\
\text { A. DC. } \\
\text { (HAMP20044) }\end{array}$ & Fagaceae & Thingsia & $\mathrm{T}$ & $\mathrm{Br}$ & Paste & $\begin{array}{l}\text { Toothache } \\
\text { (17) }\end{array}$ & 17 & 0.085 & Not reported \\
\hline 23 & $\begin{array}{l}\text { Catharanthus } \\
\text { roseus (L.) G. } \\
\text { Don } \\
\text { (HAMP20007) }\end{array}$ & Apocynaceae & Kumtluang & $\mathrm{T}$ & Lf & Decoction & $\begin{array}{l}\text { Hypertension } \\
\text { (18), diabetes } \\
(20)\end{array}$ & 38 & 0.19 & $\begin{array}{l}\text { 4-0-caffeoylquin } \\
\text { quercetin-3- 0-(6 } \\
\text { rhamnosyl-glucc } \\
\text { kaempferol-3-0- } \\
\text { rhamnosyl-glucc }\end{array}$ \\
\hline 24 & $\begin{array}{l}\text { Cheilocostus } \\
\text { speciosus (J. } \\
\text { Koenig) C.D. } \\
\text { Specht } \\
\text { (HAMP20090) }\end{array}$ & Zingiberaceae & Sumbul & Sh & $\mathrm{Rh}$ & Decoction & $\begin{array}{l}\text { Kidney disease } \\
\text { (5), urinary } \\
\text { problem }(16), \\
\text { stomach ache } \\
(18)\end{array}$ & 39 & 0.195 & $\begin{array}{l}\text { 24-hydroxytriacc } \\
\text { one, sitosterol, c } \\
\text { methyl triaconta }\end{array}$ \\
\hline
\end{tabular}

Habit: H-Herbs; Sh-Shurbs; Cr-Creeper; T-Tree UR-Used reports FC-Frequency of citation UV-Use value

bPart used: Lf-Leaf; Br-Bark; Fr-Fruit, Rh-Rhizome; St-Stem; S-Seed; WP-Whole Plants; YS-Young Shoot 


\begin{tabular}{|c|c|c|c|c|c|c|c|c|c|c|}
\hline $\begin{array}{l}\text { SI. } \\
\text { No. }\end{array}$ & $\begin{array}{l}\text { Species Name; } \\
\text { Voucher No }\end{array}$ & Family Name & Local Name & ${ }^{a}$ Habit & $\begin{array}{l}\text { bPart } \\
\text { Use }\end{array}$ & $\begin{array}{l}\text { Mode of } \\
\text { administration }\end{array}$ & Ailments \& UR & FC & UV & $\begin{array}{l}\text { Isolated Chemic } \\
\text { Compounds }\end{array}$ \\
\hline 25 & $\begin{array}{l}\text { Chromoleana } \\
\text { odorata (L.) R.M. } \\
\text { King \& H. Rob. } \\
\text { (HAMP20011) }\end{array}$ & Asteraceae & Tlangsam & $\mathrm{H}$ & Lf & $\begin{array}{l}\text { Decoction, } \\
\text { raw }\end{array}$ & $\begin{array}{l}\text { Kidney disease } \\
(18), \text { diarrhea } \\
(13), \text { stomach, } \\
\text { ache (8), } \\
\text { jaundice (14), } \\
\text { wound (34) }\end{array}$ & 87 & 0.435 & $\begin{array}{l}\text { a \& } \beta \text {-pinenes, } 1, \varepsilon \\
\beta \text {-Calacorene, } \beta \text {-( } \\
\text { ol [84] }\end{array}$ \\
\hline 26 & $\begin{array}{l}\text { Citrus } \\
\text { aurantiifolia } \\
\text { (Christm.) } \\
\text { Swingle } \\
\text { (HAMP20074) }\end{array}$ & Rutaceae & Champara & $\mathrm{T}$ & $\mathrm{Fr}$ & Juice & $\begin{array}{l}\text { Stomach } \\
\text { problem (7), } \\
\text { digestion (9) }\end{array}$ & 16 & 0.08 & $\begin{array}{l}\text { Pinene, Sabinen } \\
\text { Myrcene, Teliner }\end{array}$ \\
\hline 27 & $\begin{array}{l}\text { Citrus maxima } \\
\text { (Burm.) Merr. } \\
\text { (HAMP20075) }\end{array}$ & Rutaceae & Sertawk & Sh & $S$ & Raw & $\begin{array}{l}\text { Hypertension } \\
(36)\end{array}$ & 36 & 0.18 & $\begin{array}{l}\text { Naringenin,5,7- } \\
\text { dihydroxylcoum; } \\
\text { trihydroxyhenzer } \\
\text { xanthotoxol [90] }\end{array}$ \\
\hline 28 & $\begin{array}{l}\text { Citrus limon (L.) } \\
\text { Osbeck } \\
\text { (HAMP20076) }\end{array}$ & Rutaceae & Nimbu & $\mathrm{T}$ & $\mathrm{Fr}$ & Juice & $\begin{array}{l}\text { Stomach } \\
\text { problem (24), } \\
\text { digestion (44) }\end{array}$ & 68 & 0.34 & $\begin{array}{l}\text { Ascorbic acid, y- } \\
\text { Aminobutyric ac } \\
\text { aspartic acid, ar! }\end{array}$ \\
\hline 29 & $\begin{array}{l}\text { Clerodendrum } \\
\text { glandulosum } \\
\text { Lindl. } \\
\text { (HAMP20088) }\end{array}$ & Verbenaceae & Phuihnam & Sh & Lf & Decoction & $\begin{array}{l}\text { Hypertension } \\
\text { (44) }\end{array}$ & 44 & 0.22 & $\begin{array}{l}\text { Strongyloster, lu| } \\
\text { hentriacontane, I } \\
\text { acid, 2-pentadec } \\
\text { hexacosane, vita }\end{array}$ \\
\hline 30 & $\begin{array}{l}\text { Colocasia } \\
\text { esculenta (L.) } \\
\text { Schott } \\
\text { (HAMP20008) }\end{array}$ & Araceae & Dawl & $\mathrm{H}$ & $\begin{array}{l}\text { St } \\
\text { sap }\end{array}$ & Juice & $\begin{array}{l}\text { Vaginal } \\
\text { discharge/ } \\
\text { Lochia (2) }\end{array}$ & 2 & 0.01 & $\begin{array}{l}\text { 14a-methyl-5a-cl } \\
\text { 24-diene-3b, 7a-c } \\
\text { cyanidin 3-gluco } \\
\text { 13-trihydroxy-(E) } \\
\text { octadecenoic ac }\end{array}$ \\
\hline 31 & $\begin{array}{l}\text { Combretum } \\
\text { wallichii DC } \\
\text { (HAMP20024) }\end{array}$ & Combretaceae & Leihruisen & Sh & Sh & Raw & Tonsil (19) & 19 & 0.095 & Not reported \\
\hline 32 & $\begin{array}{l}\text { Crassocephalum } \\
\text { crepidioides } \\
\text { (Benth.) S. } \\
\text { Moore } \\
\text { (HAMP20012) }\end{array}$ & Asteraceae & Buar thau & $\mathrm{H}$ & Lf & Paste & $\begin{array}{l}\text { Wound } \\
\text { bleeding (14) }\end{array}$ & 14 & 0.07 & $\begin{array}{l}\text { (E)- } \beta \text {-farnesene, } \\
\text { humulene, cis- } \beta \text {-- } \\
\text { bulnesene [102] }\end{array}$ \\
\hline 33 & $\begin{array}{l}\text { Cucurbita } \\
\text { maxima } \\
\text { Duchesne } \\
\text { (HAMP20029) }\end{array}$ & Cucurbitaceae & Mai & $\mathrm{H}, \mathrm{Cr}$ & $S$ & Raw & $\begin{array}{l}\text { Instestinl } \\
\text { worm (15) }\end{array}$ & 15 & 0.075 & $\begin{array}{l}\text { Oleic acid, linole } \\
\text { palmitic acid, ca } \\
\text { syringic, vanillic, } \\
\text { coumaric [105] }\end{array}$ \\
\hline 34 & $\begin{array}{l}\text { Curcuma ceasia } \\
\text { Roxb. } \\
\text { (HAMP20091) }\end{array}$ & Zingiberaceae & Ailaidum & $\mathrm{H}$ & $\mathrm{Rh}$ & Raw, juice & $\begin{array}{l}\text { Stomach ache } \\
\text { (4), diarrhea } \\
\text { (18) }\end{array}$ & 22 & 0.11 & $\begin{array}{l}\text { a, } \beta \text {-pineneeucal' } \\
\text { camphor, camph } \\
\text { acid, quercetin [1 }\end{array}$ \\
\hline 35 & $\begin{array}{l}\text { Curcuma longa } \\
\text { L. (HAMP20092) }\end{array}$ & Zingiberaceae & Aieng & $\mathrm{H}$ & $\mathrm{Rh}$ & Powder, juice & $\begin{array}{l}\text { Ulcer (67), } \\
\text { diarrhea (4), } \\
\text { derma care } \\
\text { (30), stomach } \\
\text { ache (35) }\end{array}$ & 136 & 0.68 & $\begin{array}{l}\text { Curcumin, ar-turr } \\
\text { sesquiphellandre } \\
\text { curcumenol [10؟ }\end{array}$ \\
\hline 36 & $\begin{array}{l}\text { Cucumis sativus } \\
\text { L.h } \\
\text { (HAMP20030) }\end{array}$ & Cucurbitaceae & Fanghma & $\mathrm{H}, \mathrm{Cr}$ & Lf & $\begin{array}{l}\text { Decoction, } \\
\text { raw }\end{array}$ & $\begin{array}{l}\text { Malaria (8), } \\
\text { derma care } \\
(17)\end{array}$ & 25 & 0.125 & $\begin{array}{l}\text { Myristic acid, ka } \\
\text { avenasterol, palr } \\
\text { acid, alpha-linol€ } \\
\text { [111] }\end{array}$ \\
\hline 37 & $\begin{array}{l}\text { Dichrocephala } \\
\text { integrifolia (L.f.) } \\
\text { Kuntze } \\
\text { (HAMP20013) }\end{array}$ & Asteraceae & $\begin{array}{l}\text { Vawkek } \\
\text { tumtual }\end{array}$ & $\mathrm{H}$ & Lf & Decoction & $\begin{array}{l}\text { Kidney disease } \\
\text { (24) }\end{array}$ & 24 & 0.12 & $\begin{array}{l}\text { Stearic acid, stig } \\
7,22 \text {-dien-3-ol, } \\
\text { epifriedelanol, M } \\
\text { stearate, tritetrac } \\
\text { [113] }\end{array}$ \\
\hline
\end{tabular}

Habit: H-Herbs; Sh-Shurbs; Cr-Creeper; T-Tree UR-Used reports FC-Frequency of citation UV-Use value

bPart used: Lf-Leaf; Br-Bark; Fr-Fruit, Rh-Rhizome; St-Stem; S-Seed; WP-Whole Plants; YS-Young Shoot 


\begin{tabular}{|c|c|c|c|c|c|c|c|c|c|c|}
\hline $\begin{array}{l}\text { Sl. } \\
\text { No. }\end{array}$ & $\begin{array}{l}\text { Species Name; } \\
\text { Voucher No }\end{array}$ & Family Name & Local Name & ${ }^{\text {a Habit }}$ & $\begin{array}{l}\text { bPart } \\
\text { Use }\end{array}$ & $\begin{array}{l}\text { Mode of } \\
\text { administration }\end{array}$ & Ailments \& UR & FC & UV & $\begin{array}{l}\text { Isolated Chemic } \\
\text { Compounds }\end{array}$ \\
\hline 38 & $\begin{array}{l}\text { Dilllenia } \\
\text { pentagyna Roxb. } \\
\text { (HAMP20032) }\end{array}$ & Dilleniaceae & Kaihzawl & $\mathrm{T}$ & $\mathrm{Lf}, \mathrm{Br}$ & Decoction & $\begin{array}{l}\text { Diarrhea (21), } \\
\text { kidney disease } \\
\text { (1) }\end{array}$ & 22 & 0.11 & $\begin{array}{l}\text { Dillenetin, betuni } \\
\text { betulinic acid, q } \\
\text { kaempferol gluc } \\
\text { lupeol [118] }\end{array}$ \\
\hline 39 & $\begin{array}{l}\text { Dioscorea alata } \\
\text { L. (HAMP20033) }\end{array}$ & Discoreaceae & Bachhim & $\mathrm{H}, \mathrm{Cr}$ & $\mathrm{Fr}$ & Decoction & Cancer (27) & 27 & 0.135 & $\begin{array}{l}\text { Hydro-Q9 chrom } \\
\text { tocopherol-9, 1- } \\
\text { feruloylglycerol, } \\
\text { tocopherol [119] }\end{array}$ \\
\hline 40 & $\begin{array}{l}\text { Drymaria } \\
\text { cordata (L.) } \\
\text { Willd. ex Schult. } \\
\text { (HAMP20027) }\end{array}$ & Caryophyllaceae & Changkal rit & $\mathrm{H}, \mathrm{Cr}$ & Lf & $\begin{array}{l}\text { Decoction, } \\
\text { paste }\end{array}$ & $\begin{array}{l}\text { Rheumatism } \\
\text { (24), dysentery } \\
\text { (4) }\end{array}$ & 28 & 0.14 & $\begin{array}{l}\text { Stigmasterol, cel } \\
\text { [122] }\end{array}$ \\
\hline 41 & $\begin{array}{l}\text { Dysoxylum } \\
\text { excelsum } \\
\text { Blume. } \\
\text { (HAMP20051) }\end{array}$ & Meliaceae & Thingthupui & $\mathrm{T}$ & YS & Decoction & $\begin{array}{l}\text { Diarrhea (18), } \\
\text { hyper tension } \\
\text { (12) }\end{array}$ & 30 & 0.15 & $\begin{array}{l}\text { Isodauc-6-ene-1c } \\
\text { 4-epi-isodauc-6-6 } \\
\text { diol; } 4 \text {-epi-6a, } 10 \text { fof } \\
\text { dihydroxy-artabc }\end{array}$ \\
\hline 42 & $\begin{array}{l}\text { Elaeagnus } \\
\text { caudata Schltdl. } \\
\text { ex Momiy. } \\
\text { (HAMP20034) }\end{array}$ & Elaegnaceae & Sarzuk & $\mathrm{T}$ & Lf & Decoction & $\begin{array}{l}\text { Vaginal } \\
\text { discharge/ } \\
\text { Lochia (31) }\end{array}$ & 31 & 0.155 & Not reported \\
\hline 43 & $\begin{array}{l}\text { Elaeis } \\
\text { guineensis Jacq. } \\
\text { (HAMP20009) }\end{array}$ & Arecaceae & Oil palm & $\mathrm{T}$ & Oil & Oil & $\begin{array}{l}\text { Wound (1), } \\
\text { burn (1), hair } \\
\text { care (3) }\end{array}$ & 5 & 0.025 & $\begin{array}{l}\text { 3-isobutyl-2- } \\
\text { methoxypyrazin! } \\
\text { 2-acetyl-1-pyrroli } \\
\text { hexanoate; 3-me } \\
\text { xacetate [126] }\end{array}$ \\
\hline 44 & $\begin{array}{l}\text { Embelia vestita } \\
\text { Roxb. } \\
\text { (HAMP20010) }\end{array}$ & Arecaceae & Tling & Sh & Lf & Decoction & $\begin{array}{l}\text { Measles (16), } \\
\text { chickenpox } \\
(65)\end{array}$ & 81 & 0.405 & Not reported \\
\hline 45 & $\begin{array}{l}\text { Ensete glaucum } \\
\text { (Roxb.) } \\
\text { Cheesman } \\
\text { (HAMP20057) }\end{array}$ & Musaceae & Saisu & $\mathrm{H}$ & St & Decoction & $\begin{array}{l}\text { Nephrolithiasis } \\
\text { (34) }\end{array}$ & 34 & 0.17 & Not reported \\
\hline 46 & $\begin{array}{l}\text { Ensete } \\
\text { superbum } \\
\text { (Roxb.) } \\
\text { Cheesman } \\
\text { (HAMP20058) }\end{array}$ & Musaceae & $\begin{array}{l}\text { Changel/ } \\
\text { Tumbu }\end{array}$ & $\mathrm{H}$ & $\begin{array}{l}\text { St } \\
\text { sap, } \\
\mathrm{Fr}\end{array}$ & Juice & $\begin{array}{l}\text { Snake bite (6), } \\
\text { kidney disease } \\
\text { (1), diabetes } \\
\text { (4), WBC } \\
\text { deficiency (23) }\end{array}$ & 34 & 0.17 & $\begin{array}{l}\text { Pentadecanoic a } \\
\text { Pyran-4-one, 2,3- } \\
\text { 5-dihydroxy-6-mi } \\
\text { Furancarboxald } \epsilon \\
\text { (hydroxymethyl) }\end{array}$ \\
\hline 47 & $\begin{array}{l}\text { Erythrina stricta } \\
\text { Roxb. } \\
\text { (HAMP20042) }\end{array}$ & Fabaceae & Fartuah & $\mathrm{T}$ & $\mathrm{Br}$ & Decoction & $\begin{array}{l}\text { Ulcer (8), } \\
\text { kidney disease } \\
(2)\end{array}$ & 10 & 0.05 & $\begin{array}{l}\text { } \beta \text {-caryophyllene; } \\
\text { cadenine; alpinu } \\
\text { isoflavone; obov } \\
\text { isovanillin [131] }\end{array}$ \\
\hline 48 & $\begin{array}{l}\text { Eulophia nuda } \\
\text { Lindl. } \\
\text { (HAMP20062) }\end{array}$ & Orchidaceae & Nauban & $\mathrm{H}$ & WP & Decoction & Diarrhoea (2) & 2 & 0.01 & $\begin{array}{l}\text { Eulophiol;3,4-dih } \\
\text { 3,5,5-trimethoxy } \\
\text { nudol; lupeol [13 }\end{array}$ \\
\hline 49 & $\begin{array}{l}\text { Euphorbia millii } \\
\text { Des Moul. } \\
\text { (HAMP20037) }\end{array}$ & Euphorbiaceae & Hlinglukhum & $\mathrm{H}$ & Lf & Raw & Diarrhea (52) & 52 & 0.26 & $\begin{array}{l}\text { Abruquinone B; } \\
\text { eremopetasiteniı } \\
\text { acid; isopetasos } \\
\text { Dihydroxycouma }\end{array}$ \\
\hline 50 & $\begin{array}{l}\text { Euphorbia } \\
\text { royleana Boiss. } \\
\text { (HAMP20038) }\end{array}$ & Euphorbiaceae & Chawng & Sh & $\begin{array}{l}\text { Lf } \\
\text { sap }\end{array}$ & Juice & Otorrhoea (38) & 38 & 0.19 & $\begin{array}{l}\text { Antiquorine A; El } \\
\text { sandaracopimar } \\
\text { 15-isopimaradie } \\
\text { [139] }\end{array}$ \\
\hline 51 & $\begin{array}{l}\text { Flueggea virosa } \\
\text { (Roxb. ex Willd.) } \\
\text { Royle } \\
\text { (HAMP20039) }\end{array}$ & Euphorbiaceae & Saisiak & Sh & Lf & Decoction & $\begin{array}{l}\text { Diabetes (59), } \\
\text { stomach ache } \\
\text { (17), chicken } \\
\text { pox (50) }\end{array}$ & 126 & 0.63 & $\begin{array}{l}\text { 11-0-acetyl berg } \\
\text { acid; virosecurin } \\
\text { kaempferol; } \beta \text {-sit } \\
\text { quercetin [142] }\end{array}$ \\
\hline 52 & $\begin{array}{l}\text { Gomphogyne } \\
\text { cissiformis Griff. } \\
\text { (HAMP20031) }\end{array}$ & Cucurbitaceae & $\begin{array}{l}\text { Lalruanga } \\
\text { dawi bur }\end{array}$ & $\mathrm{H}, \mathrm{Cr}$ & $\mathrm{Fr}$ & Juice & $\begin{array}{l}\text { Hypertension } \\
\text { (6), diabetes } \\
\text { (7), }\end{array}$ & 13 & 0.065 & Not reported \\
\hline
\end{tabular}

Habit: H-Herbs; Sh-Shurbs; Cr-Creeper; T-Tree UR-Used reports FC-Frequency of citation UV-Use value

bPart used: Lf-Leaf; Br-Bark; Fr-Fruit, Rh-Rhizome; St-Stem; S-Seed; WP-Whole Plants; YS-Young Shoot 


\begin{tabular}{|c|c|c|c|c|c|c|c|c|c|c|}
\hline $\begin{array}{l}\text { Sl. } \\
\text { No. }\end{array}$ & $\begin{array}{l}\text { Species Name; } \\
\text { Voucher No }\end{array}$ & Family Name & Local Name & ${ }^{\text {a Habit }}$ & $\begin{array}{l}\text { bPart } \\
\text { Use }\end{array}$ & $\begin{array}{l}\text { Mode of } \\
\text { administration }\end{array}$ & Ailments \& UR & FC & UV & $\begin{array}{l}\text { Isolated Chemic } \\
\text { Compounds }\end{array}$ \\
\hline 53 & $\begin{array}{l}\text { Hedyotis } \\
\text { scandens Roxb. } \\
\text { (HAMP20072) }\end{array}$ & Rubiaceae & Kelhnam tur & $\mathrm{H}$ & Lf & Decoction & $\begin{array}{l}\text { Kidney disease } \\
\text { (4), pain relief } \\
\text { (7), diabetes } \\
\text { (10) }\end{array}$ & 21 & 0.105 & $\begin{array}{l}\text { Hedyotoside A; } \\
\text { hedyotoside B; } \\
\text { C, D \& E [145] }\end{array}$ \\
\hline 54 & $\begin{array}{l}\text { Helicia robusta } \\
\text { (Roxb.) R.Br. ex } \\
\text { Blume } \\
\text { (HAMP20069) }\end{array}$ & Protiaceae & Pasal-taka-za & $\mathrm{T}$ & Lf & Decoction & $\begin{array}{l}\text { Placenta } \\
\text { discharge (6), } \\
\text { stomach pain } \\
\text { (8), kidney } \\
\text { disorder (1) }\end{array}$ & 15 & 0.075 & Not reported \\
\hline 55 & $\begin{array}{l}\text { Hibiscus } \\
\text { sinensis Mill. } \\
\text { (HAMP20052) }\end{array}$ & Malvaceae & Midum par & Sh & Lf & Paste & Boil (4) & 4 & 0.02 & $\begin{array}{l}\text { Quercetin-3,5-di } \\
\text { undecanoic acic } \\
\text { chorides, rachid } \\
\text { cyanin [148] }\end{array}$ \\
\hline 56 & $\begin{array}{l}\text { Houttuynia } \\
\text { cordata Thunb. } \\
\text { (HAMP20077) }\end{array}$ & Saurauraceae & Ui thinthang & $\mathrm{H}$ & Lf & Juice & $\begin{array}{l}\text { Sainus } \\
\text { problem (16) }\end{array}$ & 16 & 0.08 & $\begin{array}{l}\text { Quercetin-3-0- } \beta- \\
\text { galactoside-7-O- } \\
\text { glucoside, aristo } \\
\mathrm{B}, \beta \text {-Sitosterol, } \mathrm{N} \\
\text { benzamide [150 }\end{array}$ \\
\hline 57 & $\begin{array}{l}\text { Ipomoea } \\
\text { batatas (L.) } \\
\text { Lam. } \\
\text { (HAMP20025) }\end{array}$ & Convovulaceae & Kawl-ba-hra & $\mathrm{H}, \mathrm{Cr}$ & YS & Raw & Digestion (37) & 37 & 0.185 & $\begin{array}{l}\text { 3-mono-O-caffe } \\
\text { acid; caffeic aci } \\
\text { C; kaempferol [1 }\end{array}$ \\
\hline 58 & $\begin{array}{l}\text { Lablab } \\
\text { purpureus (L.) } \\
\text { Sweet } \\
\text { (HAMP20043) }\end{array}$ & Fabaceae & Bepui & Sh & Lf & Paste & $\begin{array}{l}\text { Vaccine pain } \\
\text { relief (18) }\end{array}$ & 18 & 0.09 & $\begin{array}{l}\text { Phytic acid, lino } \\
\text { linolenic acid, [1 }\end{array}$ \\
\hline 59 & $\begin{array}{l}\text { Laurocerasus } \\
\text { undulata (Buch- } \\
\text { Ham. ex D. Don) } \\
\text { M. Roem. } \\
\text { (HAMP20071) }\end{array}$ & Rosaceae & Thei-ar-lung & $\mathrm{H}$ & Lf & Decoction & $\begin{array}{l}\text { Heart disease } \\
\text { (14) }\end{array}$ & 14 & 0.07 & Not reported \\
\hline 60 & $\begin{array}{l}\text { Lindernia } \\
\text { ruellioides } \\
\text { (Colsm.) Pennell } \\
\text { (HAMP20078) }\end{array}$ & Schrophulariaceae & Thasuih & $\mathrm{H}$ & WP & Paste & Sciatica (24) & 24 & 0.12 & $\begin{array}{l}\text { Linderruellioside } \\
\text { plantainoside A; } \\
\text { desrhamnosylve } \\
\text { [154] }\end{array}$ \\
\hline 61 & $\begin{array}{l}\text { Lobelia angulata } \\
\text { G. Forst. } \\
\text { (HAMP20020) }\end{array}$ & Campanulacea & Choak-a-thi & $\mathrm{H}$ & WP & Decoction & $\begin{array}{l}\text { Tonsil (9), } \\
\text { gallstone (16) }\end{array}$ & 25 & 0.125 & Not reported \\
\hline 62 & $\begin{array}{l}\text { Maesa indica } \\
\text { (Roxb.) A. DC. } \\
\text { (HAMP20060) }\end{array}$ & Myrsinaceae & Arngeng & Sh & Lf & Paste & Toothache (4) & 4 & 0.02 & $\begin{array}{l}25 \text { dihydroxy-6- } \\
\text { (hemeos 16-eny } \\
\text { 4benzoquinone }\end{array}$ \\
\hline 63 & $\begin{array}{l}\text { Magnolia } \\
\text { champaca (L.) } \\
\text { Baill. ex Pierre } \\
\text { (HAMP20050) }\end{array}$ & Magnoliaceae & Ngiau & $\mathrm{T}$ & Lf & Miceration & Itchy eyes (18) & 18 & 0.09 & $\begin{array}{l}\text { Butanoic acid, } 2 \\
\text { oxo-, ethyl ester; } \\
\text { Camphorsulfoni } \\
\text { [158] }\end{array}$ \\
\hline 64 & $\begin{array}{l}\text { Mangifera } \\
\text { indica L. } \\
\text { (HAMP20002) }\end{array}$ & Anacardiaceae & Theihai & $\mathrm{T}$ & YS & Decoction & $\begin{array}{l}\text { Diarrhea (5), } \\
\text { diabetes (10), } \\
\text { hypertension } \\
\text { (10), asthma } \\
(6)\end{array}$ & 31 & 0.155 & $\begin{array}{l}\text { Quercetin-3-0- } \beta- \\
\text { rhamnopyranos } \\
\text { maclurin-3-C- } \beta-\mathrm{g} \\
\text { amentoflavone; } \\
{[160]}\end{array}$ \\
\hline 65 & $\begin{array}{l}\text { Mentha arvensis } \\
\text { L. (HAMP20048) }\end{array}$ & Lamiaceae & Pudina & $\mathrm{H}$ & Lf & Decoction & $\begin{array}{l}\text { Stomach } \\
\text { problem (38) }\end{array}$ & 38 & 0.19 & $\begin{array}{l}\text { Menthol, p-ment } \\
\text { menthone, iso-m } \\
\text { [162] }\end{array}$ \\
\hline 66 & $\begin{array}{l}\text { Mesua ferrea L. } \\
\text { (HAMP20022) }\end{array}$ & Clusiaceae & Herhse & $\mathrm{T}$ & Lf & $\begin{array}{l}\text { Paste, } \\
\text { decoction }\end{array}$ & $\begin{array}{l}\text { Wound (8), } \\
\text { stomachache } \\
\text { (7), diarrhea } \\
\text { (3) }\end{array}$ & 18 & 0.09 & $\begin{array}{l}\text { 1,5-dihydroxyxa } \\
\text { euxanthone, 7-m } \\
\beta \text {-sitosterol [164 }\end{array}$ \\
\hline
\end{tabular}

Habit: H-Herbs; Sh-Shurbs; Cr-Creeper; T-Tree UR-Used reports FC-Frequency of citation UV-Use value

bPart used: Lf-Leaf; Br-Bark; Fr-Fruit, Rh-Rhizome; St-Stem; S-Seed; WP-Whole Plants; YS-Young Shoot 


\begin{tabular}{|c|c|c|c|c|c|c|c|c|c|c|}
\hline $\begin{array}{l}\text { Sl. } \\
\text { No. }\end{array}$ & $\begin{array}{l}\text { Species Name; } \\
\text { Voucher No }\end{array}$ & Family Name & Local Name & ${ }^{\text {a Habit }}$ & $\begin{array}{l}\text { bPart } \\
\text { Use }\end{array}$ & $\begin{array}{l}\text { Mode of } \\
\text { administration }\end{array}$ & Ailments \& UR & FC & UV & $\begin{array}{l}\text { Isolated Chemic } \\
\text { Compounds }\end{array}$ \\
\hline 67 & $\begin{array}{l}\text { Mikania } \\
\text { micrantha Kunth } \\
\text { (HAMP20014) }\end{array}$ & Asteraceae & Japan Hlo & $\mathrm{H}, \mathrm{Cr}$ & Lf & $\begin{array}{l}\text { Paste, } \\
\text { decoction }\end{array}$ & $\begin{array}{l}\text { Wound (52), } \\
\text { diarrhea (8), } \\
\text { dysentery (6), } \\
\text { stomach ache } \\
\text { (16) }\end{array}$ & 82 & 0.41 & $\begin{array}{l}\beta \text {-cubebene; } 1 \mathrm{H}-\mathrm{i} \\
\text { one, } 5-(1,1- \\
\text { dimethylethyl)-2, } \\
\text { aromadendrene; } \\
\text { caryophyllene [1 }\end{array}$ \\
\hline 68 & $\begin{array}{l}\text { Mimosa pudica } \\
\text { L. (HAMP20054) }\end{array}$ & Mimosaceae & Hlonuar & $\mathrm{H}$ & Lf & Decoction & $\begin{array}{l}\text { Kidney disease } \\
\text { (37) }\end{array}$ & 37 & 0.185 & $\begin{array}{l}\text { 7, 8, 3', 4'-tetrahy } \\
\text { [alpha-- L-rhamn } \\
(1-\rightarrow 2)] \text {-beta- D- } \\
\text { glucopyranosyl } \\
\text { catcher; } 5,7,3^{\prime}, 4 \\
\text { tetrahydroxyl-6-C } \\
\text { rhamnopyranosy } \\
\text { beta- D-glucopyr } \\
\text { flavone [169] }\end{array}$ \\
\hline 69 & $\begin{array}{l}\text { Morus macroura } \\
\text { Miq. } \\
\text { (HAMP20056) }\end{array}$ & Moraceae & Lung-li & $\mathrm{T}$ & Lf & Raw & $\begin{array}{l}\text { Cut / wound } \\
\text { (22) }\end{array}$ & 2 & 0.01 & $\begin{array}{l}\text { Gallic acid, catec } \\
\text { hydroxy benzoic } \\
\text { acid,3,4,5- methc } \\
\text { cinnamic [171] }\end{array}$ \\
\hline 70 & $\begin{array}{l}\text { Musa } \\
\text { acuminata Colla } \\
\text { (HAMP20059) }\end{array}$ & Musaceae & Balhla & $\mathrm{H}$ & $\begin{array}{l}\text { Fr, } \\
\text { sap }\end{array}$ & $\begin{array}{l}\text { Paste, } \\
\text { Decoction }\end{array}$ & $\begin{array}{l}\text { Diabetic } \\
\text { wound (6), } \\
\text { lung disease } \\
\text { (2), snake bite } \\
\text { (4) }\end{array}$ & 12 & 0.06 & $\begin{array}{l}\text { Pantothenic acic } \\
\text { ferulic acid-hexo } \\
\text { Vitamin C, provit } \\
\text { lycopene [172] }\end{array}$ \\
\hline 71 & $\begin{array}{l}\text { Mussaenda } \\
\text { macrophylla } \\
\text { Wall. } \\
\text { (HAMP20072) }\end{array}$ & Rubiaceae & Va-kep & Sh & Lf & Decoction & $\begin{array}{l}\text { Internal } \\
\text { bleeding (17) }\end{array}$ & 17 & 0.085 & $\begin{array}{l}\text { 3-0-B-d-glucopyr } \\
\text { O-a---rhamnopyri } \\
\text { 16a-hydroxy-23- } \\
\text { deoxyprotobassi } \\
16 \text { a-hydroxyprot } \\
\text { acid; } 3-0 \text {-acetyld } \\
\text { rotundic acid } 17\end{array}$ \\
\hline 72 & $\begin{array}{l}\text { Ocimum } \\
\text { americanum L. } \\
\text { (HAMP20049) }\end{array}$ & Lamiaceae & Runhmui & $\mathrm{H}$ & Lf, St & Paste & $\begin{array}{l}\text { Pile problem } \\
\text { (2) }\end{array}$ & 2 & 0.01 & $\begin{array}{l}\text { á-pinene; farnes } \\
\text { terpineol; farnesı } \\
\text { [177] }\end{array}$ \\
\hline 73 & $\begin{array}{l}\text { Oroxylum } \\
\text { indicum (L.) } \\
\text { Kurz } \\
\text { (HAMP20018) }\end{array}$ & Bignoniaceae & $\begin{array}{l}\text { Ar-chang- } \\
\text { kawm }\end{array}$ & $\mathrm{T}$ & $\mathrm{Br}, \mathrm{Lf}$ & $\begin{array}{l}\text { Paste, } \\
\text { decoction }\end{array}$ & $\begin{array}{l}\text { Ulcer (6), } \\
\text { arthritis (13), } \\
\text { diarrhea (3), } \\
\text { dysentry (6), } \\
\text { hepatitis C (9) }\end{array}$ & 37 & 0.185 & $\begin{array}{l}\text { Ellagic acid; oro> } \\
\text { methyl chrysin; } \mathrm{F} \\
\text { linoleic acids [1 }\end{array}$ \\
\hline 74 & $\begin{array}{l}\text { Pandanus } \\
\text { odorifer } \\
\text { (Forssk.) Kuntze } \\
\text { (HAMP20064) }\end{array}$ & Pandanaceae & Ram-la-kuih & Sh & Lf & Decoction & $\begin{array}{l}\text { Kidney disease } \\
24\end{array}$ & 24 & 0.12 & Not reported \\
\hline 75 & $\begin{array}{l}\text { Parkia timoriana } \\
\text { (DC.) Merr. } \\
\text { (HAMP20055) }\end{array}$ & Mimosaceae & Zawngtah & $\mathrm{T}$ & $\begin{array}{l}\mathrm{Fr}, \\
\text { peel, } \\
\mathrm{Br}\end{array}$ & Paste, raw & $\begin{array}{l}\text { Diabetes (10), } \\
\text { baby umblical } \\
\text { care }(2), \\
\text { hypertension } \\
\text { (4) }\end{array}$ & 16 & 0.08 & $\begin{array}{l}\beta \text {-sitostero; javar } \\
\text { epigallocatechin } \\
\text { ursolic acid; hyp }\end{array}$ \\
\hline 76 & $\begin{array}{l}\text { Phylanthus } \\
\text { emblica L. } \\
\text { (HAMP20065) }\end{array}$ & Phyllanthaceae & Sunhlu & $\mathrm{T}$ & $\mathrm{Fr}$ & Raw, juice & $\begin{array}{l}\text { Diarrhea (2), } \\
\text { skincare (12), } \\
\text { stomach } \\
\text { problem (8), } \\
\text { energybooster } \\
\text { (7), diabetes } \\
(9), \\
\text { hypertension } \\
\text { (6), jaundice } \\
(4)\end{array}$ & 48 & 0.24 & $\begin{array}{l}\text { Ascorbic acids, } c \\
\text { quercetin, punigl } \\
\text { emblicanin A\&B, } \\
\text { acids [184] }\end{array}$ \\
\hline 77 & $\begin{array}{l}\text { Picria fel-terrae } \\
\text { Lour. } \\
\text { (HAMP20079) }\end{array}$ & Scrophulariaceae & Khatual & $\mathrm{H}$ & Lf & Decoction & $\begin{array}{l}\text { Hypertension } \\
\text { (24) }\end{array}$ & 24 & 0.12 & $\begin{array}{l}\text { Rosmarinic acid } \\
7-0-\beta \text {-D-glucuron } \\
\text { picfeltarraegenir } \\
\text { acteoside; apige }\end{array}$ \\
\hline 78 & $\begin{array}{l}\text { Plantago major } \\
\text { L. (HAMP20066) }\end{array}$ & Plantaginaceae & Kelba-an & $\mathrm{H}$ & Lf & Decoction & $\begin{array}{l}\text { Kidney disease } \\
\text { (2), urinary } \\
\text { problem (34) }\end{array}$ & 36 & 0.18 & $\begin{array}{l}\text { Plantaginin; plar } \\
\text { ursolic acid; auc } \\
\text { palmitic acid, as } \\
{[188]}\end{array}$ \\
\hline
\end{tabular}

Habit: H-Herbs; Sh-Shurbs; Cr-Creeper; T-Tree UR-Used reports FC-Frequency of citation UV-Use value

bPart used: Lf-Leaf; Br-Bark; Fr-Fruit, Rh-Rhizome; St-Stem; S-Seed; WP-Whole Plants; YS-Young Shoot 


\begin{tabular}{|c|c|c|c|c|c|c|c|c|c|c|}
\hline $\begin{array}{l}\text { SI. } \\
\text { No. }\end{array}$ & $\begin{array}{l}\text { Species Name; } \\
\text { Voucher No }\end{array}$ & Family Name & Local Name & a Habit & $\begin{array}{l}\text { bPart } \\
\text { Use }\end{array}$ & $\begin{array}{l}\text { Mode of } \\
\text { administration }\end{array}$ & Ailments \& UR & FC & UV & $\begin{array}{l}\text { Isolated Chemic: } \\
\text { Compounds }\end{array}$ \\
\hline 79 & $\begin{array}{l}\text { Psidium guajava } \\
\text { L. (HAMP20061) }\end{array}$ & Myrtaceae & Kawl-thei & $\mathrm{T}$ & Lf & Decoction & $\begin{array}{l}\text { Diarrhea (97), } \\
\text { hair fall (1) }\end{array}$ & 98 & 0.49 & $\begin{array}{l}\beta \text {-sitosterol; guaj } \\
\text { oleanolic acid; u' } \\
\text { ursolic acid [189 }\end{array}$ \\
\hline 80 & $\begin{array}{l}\text { Pseudodrynaria } \\
\text { coronans (Wall. } \\
\text { ex Mett.) Ching } \\
\text { (HAMP20067) }\end{array}$ & Polypodiaceae & Awmvel & $\mathrm{H}$ & Lf & Paste & Herpes (12) & 12 & 0.06 & 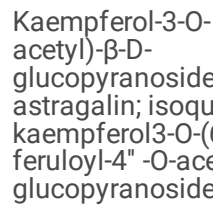 \\
\hline 81 & $\begin{array}{l}\text { Punica } \\
\text { granatum L. } \\
\text { (HAMP20068) }\end{array}$ & Punicaceae & Thei-buh-fai & $\mathrm{T}$ & $\mathrm{Fr}$ & Raw, juice & $\begin{array}{l}\text { Anemia (5), } \\
\text { immuno- } \\
\text { booster (21), } \\
\text { cancer (5) }\end{array}$ & 31 & 0.155 & $\begin{array}{l}\text { Ellagic acid; kaeı } \\
\text { anthocyanins; pı } \\
\text { quercetin; luteoli } \\
\text { ellagitannins [19 }\end{array}$ \\
\hline 82 & $\begin{array}{l}\text { Rhus chinensis } \\
\text { Mill. } \\
\text { (HAMP20003) }\end{array}$ & Anacardiaceae & Khawmhma & $\mathrm{T}$ & $\mathrm{Fr}$ & Raw, powder & $\begin{array}{l}\text { Diarrhea (27), } \\
\text { stomach ache } \\
\text { (18) }\end{array}$ & 45 & 0.225 & $\begin{array}{l}\text { Moronic acid; ga } \\
\text { oxo-6 } 6 \text {-hydroxyo } \\
\text { en-28-oic; aciddi } \\
\text { caffic acid; phytc }\end{array}$ \\
\hline 83 & $\begin{array}{l}\text { Sarcococca } \\
\text { pruniformis } \\
\text { Lindl. } \\
\text { (HAMP20040) }\end{array}$ & Euphorbiacea & Pawhrual & $\mathrm{H}$ & Lf & $\begin{array}{l}\text { Raw, } \\
\text { decoction }\end{array}$ & Tonsil (49) & 49 & 0.245 & Not reported \\
\hline 84 & $\begin{array}{l}\text { Schima wallichii } \\
\text { Choisy } \\
\text { (HAMP20083) }\end{array}$ & Theaceae & Khiang & $\mathrm{T}$ & $\mathrm{Br} / \mathrm{St}$ & Paste & $\begin{array}{l}\text { Insect bite (9), } \\
\text { wound (5) }\end{array}$ & 14 & 0.07 & $\begin{array}{l}\text { Phenylpropanola } \\
\text { rotenone; glycidc } \\
\text { benzofurandion }\end{array}$ \\
\hline 85 & $\begin{array}{l}\text { Senecio } \\
\text { scandens Buch- } \\
\text { Ham. ex D. Don } \\
\text { (HAMP20015) }\end{array}$ & Asteraceae & Sai-ek-hlo & $\mathrm{H}, \mathrm{Cr}$ & Lf & $\begin{array}{l}\text { Decoction, } \\
\text { paste }\end{array}$ & $\begin{array}{l}\text { Ulcer (17), } \\
\text { diabetes (13), } \\
\text { Hypertension } \\
\text { (8), toothache } \\
(3), \text { wound (2) }\end{array}$ & 43 & 0.215 & $\begin{array}{l}\text { Quercetin; kaem। } \\
\text { luteolin; rutin; ph } \\
\text { palmitic acid; } \beta-\text { - } \\
\text { Sitosterol [197] }\end{array}$ \\
\hline 86 & $\begin{array}{l}\text { Smilax globra } \\
\text { Roxb. } \\
\text { (HAMP20081) }\end{array}$ & Smilacaceae & Kai-tluang & $\mathrm{H}, \mathrm{Cr}$ & Lf & Paste & Arthritis (20) & 20 & 0.1 & $\begin{array}{l}\text { Palmitic acid; } \beta-\varsigma \\
\text { quercetin; apiger } \\
\text { methoxygallic ac } \\
\text { lignoceric acid [1 }\end{array}$ \\
\hline 87 & $\begin{array}{l}\text { Smilax } \\
\text { perfoliata Lour. } \\
\text { (HAMP20080) }\end{array}$ & Smilacaceae & Kai-ha & $\mathrm{Sh}, \mathrm{Cr}$ & Lf & Decoction & Dysentry (6) & (6) & 0.03 & $\begin{array}{l}\text { Rutin, } 1 ; \text {; 6-0-difer } \\
\text { p-coumaroyl)-b-[ } \\
\text { fructofuranosyl-1 } \\
\text { acetyl-a-D- } \\
\text { glucopyranoside } \\
\text { cassiamin A \& B } \\
\text { [199] }\end{array}$ \\
\hline 88 & $\begin{array}{l}\text { Solanum } \\
\text { americanum } \\
\text { Mill. } \\
\text { (HAMP20085) }\end{array}$ & Solanaceae & Anhling & Sh & Lf & Decoction & $\begin{array}{l}\text { Nephrolithiasis } \\
\text { (15), urinary } \\
\text { retention (6), } \\
\text { Kidney disease } \\
(11)\end{array}$ & 32 & 0.16 & $\begin{array}{l}\text { Pinoresinol; tetra } \\
\text { acid; syringaresi } \\
\text { sitosterol; scopo } \\
\text { medioresinol [20 }\end{array}$ \\
\hline 89 & $\begin{array}{l}\text { Solanum } \\
\text { violaceum } \\
\text { Ortega } \\
\text { (HAMP20086) }\end{array}$ & Solanaceae & Tawkte & Sh & $\mathrm{Fr}$ & $\begin{array}{l}\text { Decoction, } \\
\text { paste }\end{array}$ & $\begin{array}{l}\text { Hypertension } \\
(20) \text {, diabetes } \\
(6) \text {, burn (4), } \\
\text { boil (1), herpes } \\
(2)\end{array}$ & 33 & 0.165 & $\begin{array}{l}\text { 7-oxositosterol; ) } \\
\text { 7- } \\
\text { oxostigmasterol } \\
{[204]}\end{array}$ \\
\hline 90 & $\begin{array}{l}\text { Spondias } \\
\text { pinnata (L. f.) } \\
\text { Kurz } \\
\text { (HAMP20004 }\end{array}$ & Anacardiaceae & Tawi-taw & $\mathrm{T}$ & $\mathrm{Br}$ & Decoction & Diarrhea (3) & 3 & 0.015 & $\begin{array}{l}\text { Ellagitannins, } \\
\text { galloylgeranin, li } \\
\text { acid, } \beta \text {-carotein, } \\
\text { acid [208] }\end{array}$ \\
\hline
\end{tabular}

Habit: H-Herbs; Sh-Shurbs; Cr-Creeper; T-Tree UR-Used reports FC-Frequency of citation UV-Use value

bPart used: Lf-Leaf; Br-Bark; Fr-Fruit, Rh-Rhizome; St-Stem; S-Seed; WP-Whole Plants; YS-Young Shoot 


\begin{tabular}{|c|c|c|c|c|c|c|c|c|c|c|}
\hline $\begin{array}{l}\text { Sl. } \\
\text { No. }\end{array}$ & $\begin{array}{l}\text { Species Name; } \\
\text { Voucher No }\end{array}$ & Family Name & Local Name & aHabit & $\begin{array}{l}\text { bPart } \\
\text { Use }\end{array}$ & $\begin{array}{l}\text { Mode of } \\
\text { administration }\end{array}$ & Ailments \& UR & FC & UV & $\begin{array}{l}\text { Isolated Chemic } \\
\text { Compounds }\end{array}$ \\
\hline 91 & $\begin{array}{l}\text { Tectona grandis } \\
\text { L.f. } \\
\text { (HAMP20089) }\end{array}$ & Verbenaceae & Teak & $\mathrm{T}$ & $\mathrm{Br}, \mathrm{Lf}$ & Paste & $\begin{array}{l}\text { Wound } \\
\text { bleeding (2) }\end{array}$ & 2 & 0.01 & $\begin{array}{l}\text { Gallic acid; } \beta \text {-sitc } \\
\text { betulinic acid; } \\
\text { tectoquinone; sq } \\
\text { lauric acid [209] }\end{array}$ \\
\hline 92 & $\begin{array}{l}\text { Zea mays L. } \\
\text { (HAMP20070) }\end{array}$ & Poaceae & Vaimim & $\mathrm{H}$ & Lf & Decoction & $\begin{array}{l}\text { Kidney disease } \\
\text { (15) }\end{array}$ & 15 & 0.075 & $\begin{array}{l}\text { Eugenol; cis-a-te } \\
\text { citronellol; 6,11-c } \\
\text { 4-ene [211] }\end{array}$ \\
\hline 93 & $\begin{array}{l}\text { Zingiber } \\
\text { officinale } \\
\text { Roscee } \\
\text { (HAMP20093) }\end{array}$ & Zingiberaceae & Sawhthing & $\mathrm{H}$ & $\mathrm{Rh}$ & $\begin{array}{l}\text { Raw, } \\
\text { decoction }\end{array}$ & $\begin{array}{l}\text { Cold / cough } \\
\text { (12), digestion } \\
\text { (18) }\end{array}$ & 30 & 0.15 & $\begin{array}{l}\text { Zingiberene; gine } \\
\text { farnesene; curcu } \\
\text { zingerone; vitam }\end{array}$ \\
\hline
\end{tabular}

Habit: H-Herbs; Sh-Shurbs; Cr-Creeper; T-Tree UR-Used reports FC-Frequency of citation UV-Use value

bPart used: Lf-Leaf; Br-Bark; Fr-Fruit, Rh-Rhizome; St-Stem; S-Seed; WP-Whole Plants; YS-Young Shoot

\section{Usage analysis based on the treatment of ailments}

The total number of user reports documented in this study was 2717, in which all different illnesses were categorised into 16 groups. Among the illness category, the gastro-intestinal disease has the highest usage report (940) followed by skincare (259) cardiovascular (222), kidney disease (196), hyperglycaemia (175), ENT (159), genito-urinary disease (139) and so on as shown in (Table 4). 
Usage analysis with illness category and their term.

\begin{tabular}{|c|c|c|c|}
\hline $\begin{array}{l}\text { Illness } \\
\text { Categories }\end{array}$ & $\begin{array}{l}\text { Medical } \\
\text { Term }\end{array}$ & Local Term & $\begin{array}{l}\text { Frequency of } \\
\text { usage }\end{array}$ \\
\hline $\begin{array}{l}\text { Dental Care } \\
\text { (DC) }\end{array}$ & $\begin{array}{l}\text { Tooth decay } \\
\text { Toothpaste }\end{array}$ & $\begin{array}{l}\text { Ha muat / Ha nget } \\
\text { Ha nawhna }\end{array}$ & 57 \\
\hline $\begin{array}{l}\text { Skin Care } \\
\text { (SC) }\end{array}$ & $\begin{array}{l}\text { Pimple, burn, } \\
\text { Face pack, boil, chickenpox, Measles, Herpes, }\end{array}$ & $\begin{array}{l}\text { Bawl, Kang, Sentut, Khawihli, } \\
\text { Awmvel, Tangseh } \\
\text { Hmai chiahna, }\end{array}$ & 259 \\
\hline Hair Care $(\mathrm{HC})$ & $\begin{array}{l}\text { Growth enhances, } \\
\text { shining, hair fall }\end{array}$ & $\begin{array}{l}\text { Sam thatna, Sam tletna, Sam } \\
\text { tla }\end{array}$ & 16 \\
\hline $\begin{array}{l}\text { Eyes/Nose/ } \\
\text { Ears/Mouth } \\
\text { (ENT) }\end{array}$ & Otorrhoea, eye itching, Sinusitis, Tonsilitis & $\begin{array}{l}\text { Beng kherh, Mit thak, Sinus, } \\
\text { Tonsil }\end{array}$ & 159 \\
\hline $\begin{array}{l}\text { Genito-urinary } \\
\text { Disease (GUD) }\end{array}$ & Delivery pain, placenta discharge, urine retention, & $\begin{array}{l}\text { Nau neih zawh hlam tlakna / } \\
\text { na, } \\
\text { Zun in }\end{array}$ & 139 \\
\hline $\begin{array}{l}\text { Kidney Disease } \\
\text { (KD) }\end{array}$ & Nephrolithiasis, kidney failure & $\begin{array}{l}\text { Kal a lungte awm, Kalna, Kal a } \\
\text { hnai awm }\end{array}$ & 196 \\
\hline $\begin{array}{l}\text { High glucose level } \\
(\mathrm{HGL})\end{array}$ & Diabetes type I \& II & Zunthlum & 175 \\
\hline $\begin{array}{l}\text { Cancer Disease } \\
\text { (CD) }\end{array}$ & Breast cancer, Leukemia, lung cancer, etc & $\begin{array}{l}\text { Hnute Cancer, Thisen cancer, } \\
\text { Chuap cancer leh dangte }\end{array}$ & 75 \\
\hline $\begin{array}{l}\text { Liver Problem } \\
\text { (LP) }\end{array}$ & $\begin{array}{l}\text { Jaundice, Hepatitis B \& C, cholelithiasis, } \\
\text { Malaria }\end{array}$ & $\begin{array}{l}\text { Thinlian, Hepatitis, Malaria, } \\
\text { Mit a lungte awm }\end{array}$ & 100 \\
\hline $\begin{array}{l}\text { Cardiovascular } \\
\text { Problem (CP) }\end{array}$ & Hypertension, Heart problem & Bp sang leh hniam, lung thalo & 222 \\
\hline $\begin{array}{l}\text { Muscular / Bone } \\
\text { Problem (MBP) }\end{array}$ & Rheumatoid, Arthritis, Sciatica & Ruh seh/Sehpui, thana, Scaitica & 81 \\
\hline $\begin{array}{l}\text { Respiratory System } \\
\text { illness (RSI) }\end{array}$ & Cold, Cough, Asthma, lung disease & $\begin{array}{l}\text { Hritlang, Awmna, Khuh, } \\
\text { Thawhah }\end{array}$ & 42 \\
\hline $\begin{array}{l}\text { Gastro-intestinal } \\
\text { Disease (GID) }\end{array}$ & $\begin{array}{l}\text { Ulcer, stomach pain, Dysentry, Diarrhea, Digestion, Hemorrhoid, Constipation, } \\
\text { Intestinal worms, internal bleeding }\end{array}$ & $\begin{array}{l}\text { Ulcer, Pumna, Ek khal, Pile na, } \\
\text { Puar nuamlo, Khawthalo, } \\
\text { Santen, Rul hlut, Internal } \\
\text { bleeding }\end{array}$ & 940 \\
\hline Wound healing $(\mathrm{WH})$ & Inflammation & $\begin{array}{l}\text { Pem thar /Pilh damdawi, Pan, } \\
\text { vung }\end{array}$ & 126 \\
\hline $\begin{array}{l}\text { Poisonous Bites } \\
\text { (PB) }\end{array}$ & Snake bite, Scorpion bite, dog bite, Wasp sting & $\begin{array}{l}\text { Rul chuk, Ui seh, Khuai zuk, } \\
\text { Khawmual kaikuang seh }\end{array}$ & 45 \\
\hline General Health (GH) & Fever, Headache, cold, Immuno-booster, Energy booster, etc & $\begin{array}{l}\text { Luna, Khawsik, Taksa chakna, } \\
\text { Hriselna }\end{array}$ & 81 \\
\hline & & Total & 2717 \\
\hline
\end{tabular}

\section{Data analysis}

\section{Frequency of citation}

Among the total number of user reports (UR) cited, Curcuma longa L. (136 FC), Flueggea virosa (Roxb. ex Willd.) Royle (126 FC), Psidium guajava L. (98 FC), Chromoleana odorata (L.) R.M. King \& H. Rob. (87 FC), Mikania micrantha Kunth (82 FC), Citrus limon (L.) Osbeck (68 FC), Carica papaya L. (53 FC), Ananas comosus (L.) Merr. (49 FC), Sarcococca pruniformis Lindl. (49 FC), Phylanthus emblica L. (48 FC), Rhus chinensis Mill. (45 FC), Clerodendrum glandulosum Lindl. (44 FC), Senecio scandens Buch- Ham. ex D. Don (43 FC) were those species having the highest FC (Table 3). 


\section{Plant use value}

From the UV value evaluation, Curcuma longa L. (0.68), Flueggea virosa (Roxb. ex Willd.) Royle (0.63), Psidium guajava L. (0.49), Chromoleana odorata (L.) R.M. King \& H. Rob. (0.43), Mikania micrantha Kunth (0.41), Citrus limon (L.) Osbeck (0.34), Carica papaya L. (0.26), Ananas comosus (L.) Merr. (0.24), Sarcococca pruniformis Lindl. (0.24), Phylanthus emblica L. (0.24), Cleradendrum glandulosum Lindl. (0.22), Rhus chinensis Mill. (0.22), Senecio scandens Buch- Ham. ex D. Don (0.21) were reported to have the highest use value (UV).

\section{Informant Consensus Factor}

We calculated the informant consensus factor by categorising the reported illness into 16 ailment groups along with the number of users report and taxa as shown in (Table 5). In our study $F_{i c}$ values ranged from 0.866 to 0.962 which were all close to 1 . $F_{\text {ic }}$ value with 1 or either close to 1 indicates that a large number of informants had agreed on using few plants for curing an illness category while low $F_{\text {ic }}$ value signified that there was an argument on using medicinal plants to treat illness amidst the category.

Table 5

Informant consensus factor with their used report in each of an ailment category.

\begin{tabular}{|llll|}
\hline Illness Categories & $\begin{array}{l}\text { No of used } \\
\text { report }\end{array}$ & $\begin{array}{l}\text { No of } \\
\text { taxa }\end{array}$ & $\mathrm{F}_{\text {ic }}$ \\
\hline Dental Care (DC) & 57 & 8 & 0.875 \\
\hline Skin Care (SC) & 259 & 13 & 0.953 \\
\hline Hair Care (HC) & 16 & 3 & 0.866 \\
\hline Eyes/Nose/ Ears/Mouth (ENT) & 159 & 9 & 0.949 \\
\hline Genito-urinary Disease (GUD) & 139 & 9 & 0.942 \\
\hline Kidney Disease (KD) & 196 & 15 & 0.928 \\
\hline Endocrinal Disorder (ED) & 175 & 12 & 0.936 \\
\hline Cancer Disease (CD) & 75 & 5 & 0.945 \\
\hline Liver Problem (LP) & 100 & 7 & 0.939 \\
\hline Cardiovascular Problem (CP) & 222 & 14 & 0.941 \\
\hline Muscle/Bone Problem (MBP) & 81 & 4 & 0.962 \\
\hline Respiratory System illness (RSI) & 42 & 5 & 0.902 \\
\hline Gastro-intestinal Disease (GID) & 940 & 42 & 0.956 \\
\hline Wound healing (WH) & 126 & 10 & 0.928 \\
\hline Poisonous Bites & 45 & 5 & 0.909 \\
\hline (PB) & 81 & 10 & 0.887 \\
\hline General Health (GH) & 2717 & & \\
\hline
\end{tabular}

\section{Correlation and Validation studies}

An attempt was made to compare the use of all the medicinal plants reported by the informants with the previous papers published for their biological activity or ethnomedicines (Table 3). According to the studies conducted by Cakilcioglu et al., 2011, it was stated that if a medicinal plant has been reported for similar use in other parts of the world, its pharmacological effect could be more easily known [216].

The use of crude juice of Allium cepa L. showed a significantly higher hair growth rate than tap water when applied twice a day for two months which corroborated the present report of hair regrowth [22]. Metallothionein, an antioxidant protein present in Aloe vera (L.) gel has been reported to have a protective effect against UV and gamma radiation damage to the skin. It scavenges free radicals by preventing the suppression of glutathione peroxidase and superoxide dismutase in the skin [30]. So, this validated the used of $A$. vera for skin care and burning by the Mizo tribes. In the present study, Betula alnoides Buch- Ham. ex D. Don has been used as toothpaste for whitening teeth while it was proved that $80 \%$ methanolic bark extract had the potential a-glucosidase inhibitory effect that prevent the $(98.4 \%)$ at $40 \mu \mathrm{g} / \mathrm{mL}$ concentration [2]. Cajanus cajan (L.) Millsp is used effectively in Champhai district to treat jaundice and intestinal worms. To certify this, the methanolic extracts showed hepatoprotective activity in Swiss albino mice by inducing carbon tetrachloride ( $\left.\mathrm{CCl}_{4}\right)$ that cause liver damage. It lowers the serum levels of glutamate pyruvate transaminase (SGPT), or alanine aminotransferase (ALT) aspartate aminotransferase (AST) or serum glutamate oxaloacetate transaminase (SGOT) significantly [61]. 
When the aqueous extracts of Carica papaya L. and Ananas comosus L. were given to Spraque Dawley rats orally at doses of 5 and $10 \mathrm{mg} / \mathrm{kg}$. Both possessed mild to strong diuretic activity. Careful measure should be taken when using these plants as increase in the level of urinary $\mathrm{K}^{+}$, serum $\mathrm{BUN}$ and creatinine were mentioned [75]. This validated the used of $C$. papaya and $A$. comosus in kidney disease and urinary infection. The contemporary reports showed that Drymaria cordata (L.) was used as an instant pain killer for rheumatism meanwhile the scientific study also demonstrated that the aqueous whole plant extract exhibited analgesic and antipyretic properties at doses of 100,200 , and $400 \mathrm{mg} / \mathrm{kg}$ p.o mediated through peripheral and central mechanisms [217]. The latex water-soluble fraction of Euphorbia royleana Boiss. showed dose-dependent anti-arthritic and anti-inflammatory effects in rats and mice administered through gavage at doses of $50-200 \mathrm{mg} / \mathrm{kg}$ having more than $1500 \mathrm{mg} / \mathrm{kg}$ oral LD 50 in both [141]. Dose-dependent and significant decline in the number of abdominal constrictions induced by intraperitoneal administration of acetic acid were observed in methanol extract of $L a b l a b$ purpureus (L.) Sweet. at a dose of $200 \mathrm{mg}$ and $400 \mathrm{mg}$ exhibit far better analgesic activity than $200 \mathrm{mg}$ aspirin per kg of body weight [218].

Colocasia esculenta (L.) Schott and Elaeagnus caudata Schltdl. ex Momiy. were declared to use to discharge placenta after birth and to treat vaginal discharge (Lochia) for women in present study. Besides this record, in Cachar hills district of Assam, India $5 \mathrm{ml}$ of Elaeagnus caudata fresh root extract diluted in $10 \mathrm{ml}$ of fresh water was also administered orally once a week to prevent miscarriage during pregnancy although there is no scientific study to backup this claim [124]. Apart from present report in Jamaica, Mikania micrantha Kunth. was most popularly used too for wound healing and its extract showed antiinflammatory and antimicrobial activity against common pathogens, namely Escherichia coli, Staphylococcus aureus and Streptococcus sp [219]. The decoction of Psidium guajava leaf was effectively being used for diarrhoea which already proved to have antidiarrheal and protein conservative effects in diarrheal rats at a dose of 50 and $100 \mathrm{mg} / \mathrm{kg}$ of body weight. It increased the kidney weight and concentration of sodium, potassium and chloride significantly [220]. In the animal study of anti-urolithialic activity of Solanum nigrum, the fruit hydroalcoholic extract elicited potent activity against calcium oxalate urolithiasis effected by ethylene glycol through tumour necrosis factor adiponectin stimulation and alpha inhibition, also maintained the balance between stone promoter as well as inhibitor such as calcium and magnesium respectively [221]. Thus, this authenticated the used of $S$. nigrum for removing/forming kidney stone by the Mizo tribes in India.

Anoectochilus brevilabris Lindl., Begonia inflata C.B. Clark, Dysoxylum excelsum Blume, Embelia vestita Roxb, Ensete glaucum (Roxb.) Cheesman, Gomphogyne cissiformis Griff., Helicia robusta (Roxb.) R. Br. ex Blume, Laurocerasus undulata (Buch- Ham. ex D. Don) M. Roem. and Lobelia angulata G. Forst., Sarcococca pruniformis Lindl. were the plants that did not have biological activity reported previously which means that there is no scientific validation to support their application. Therefore, these plants were especially recommended in carrying out further investigation.

In addition, we compiled the secondary metabolite isolated chemical constituents done by the several researchers for all the documented plants in the present study. Further investigation revealed that secondary metabolites from 13 plant species that have neither less nor none chemical compound isolated or identified - Anoectochilus brevilabris Lindl., Begonia inflata C. B. Clark., Castanopsis tribuloides (Sm.) A. DC., Combretum wallichii DC, Elaeagnus caudata Schltdl. ex Momiy., Embelia vestita Roxb., Ensete glaucum (Roxb.) Cheesman, Gomphogyne cissiformis Griff., Helicia robusta (Roxb.) R. Br. ex Blume, Laurocerasus undulata (Buch- Ham. ex D. Don) M. Roem., Lobelia angulata G. Forst., Pandanus odorifer (Forssk.) Kuntze, Sarcococca pruniformis Lindl. (Table 3) which will surely have great potent on ethnopharmacological study.

\section{Discussion}

According to the finding of other workers regarding this demography of informants, the males played more role than females in ethnomedicinal practices [222, 223] which correspond to our results that women practitioners (44\%) were less than men (56\%) as being a traditional healer in the study area as shown in (Table 1). Further, the results of the usage of plants dominated by the families were followed and confirmed the work done by some researchers stating that greater the plants grew in the study area the more it will be favourably and commonly used [224]. In the present study we laid out the only accepted botanical names by 'The Plant List' and their family, local name, habit, mode of preparation and ailments as illustrated in Table 3.

The frequent use of herbaceous plants as medicines among the informants was due to their richness, abundance as well as their ability to grow easily in nature. Meanwhile, many parts of the world have been commonly using herbs as their medicinal ingredients due to their wide range of medicinal properties [225]. Leaves are the most utilised part of the plants due to their ease off collection as compared to their underground part. It is also the active site of photosynthesis accompanied by the production of metabolites $[1,226]$. In addition, leaves can be easily prepared and stored. It can be dried quickly under the sun in lesser amount of time than other parts like stem, bark and rhizome.

Similarly, it is also reported that decoction was the most common preparation method for herbal medicine while in some other tribal community [3], preparation of paste was the most common method applied $[1,223,227]$. For decoction the plant part was washed thoroughly and boiled with water administering the juice orally whereas for paste the materials were crushed or rubbed within palms and applied topically. To make fine powder plant parts were shade dried and ground. Intake of oral administration and external topical formulation were the main mode of administration used in traditional herbal medicines which has also been previously reported [222, 228]. Regarding the duration of consumption of herbal medicine, it depends on the illness whether it was short term or long term. For instance, short term illness like cold, flu, stomach upset and skin problem, the consumption period did not last more than 1 week. On the contrary, the long term illness like diabetes, kidney failure and heart diseases, the consumption period of plants (eg: Flueggea virosa) was much longer and last more than a month and so on.

From this study it was clear that among the informants, stomach problems like ulcer, indigestion, diabetes, hypertension and kidney problems were common illness resulting in high user rate of consuming herbal medicines and similar record was reported by Mahwasane et al., [229]. Further, skin problem like dermatitis which was the second highest usage report was the highest ailment in most other tribal communities like Malda district in West Bengal reported by Saha et al. [230]. 
Generally, majority of the informants did not consume the medicines prescribed by the Doctor's prescribed medicines along with their herbal medicine and claimed that many plants like Sarcococca pruniformis (tonsil), Psidium guajava (diarrhoea), Mikania micrantha (cut/wound), Flueggea virosa (chicken pox), Elaeagnus caudata (veginal discharge) were really effective and most importantly, none of them reported any adverse effect of the herbal medicines.

Moreover, regarding the expenditure on buying medicines, $38 \%$ of the informants usually purchased their herbal medicines either in raw form (Allium sativum, Allium cepa, Beta vulgaris) or in processed form like juice (Citrus limon, Phyllanthus emblica, Citrus aurantiifolia), fruits (Punica granatum, Phylanthus eamblica, Cucumis sativus), and powder (Curcuma longa). Concerned about the source of their knowledge, all the informants reported that they have heard and learned some of their information from their elders, family and/or acquaintances. Besides these, $30 \%$ of the informants have also gathered additional information through social media and $10 \%$ through books, magazines and newspapers. This documentation clearly showed that knowledge and cultural practices of herbal medicines had been shared through the indigenous community through word of mouth.

Frequency of citation showed the sociocultural importance of medicinal plants to identify their therapeutic value [17]. The FC value is directly proportional to the use value (UV), the more FC value will increase the used value significantly.

Curcuma longa L. is one of the main commercially grown as seasoning plants in India. In Southeast Asia including India and China, turmeric powder has been used extensively for spice and colouring food material. It had a wide range of medicinal value that curcumin was the main bioactive chemical constituents [231]. C. longa was a mandatory spice that each and every household kept it which/that is why the reason used report (UR) for medicinal value is high among the informants. The informants in present study reported that Flueggea virosa have a prominent effectiveness against diabetes (59 UR) and chickenpox (50 UR). The Mizo tribes extensively used F. virosa and Embelia vestita Roxb. plant to treat chickenpox and measles by bathing once a day with the decoction of leaf mixed with water.

Higher in the UV value indicates the more rate of agreeing and sharing their knowledges and practices of the medicinal plants among the informants [223]. Among the Terai forest of western Nepal Curcuma longa L. was also reported as the highest used value [232] similar to this result.

The plants with low UV value were Colocasia esculenta (L.) Schott, Eulophia nuda Lindl and Ocimum americanum L., Maesa indica (Roxb.) A. DC, Morus macroura Miq, Tectona grandis L.f., Hibiscus sinensis Mill, Eleais guineensis Jacq, Smilax perfoliata Lour with less than 0.05 UV as shown in (Table 3). Tectona grandis L.f. was also described with very low UV value by Ayyanar and Ignacimuthu as relevant to this result [1]. According to Chaudhary et al. 2006, the plants with low used value were in at risk of misrecollecting and passing on to the young generation which might be gradually disappearing [233]. On the other hand, the relevance of knowing the plant used value was for the convenience of pharmacological study and their used reliability [234].

However, Rajakumar and Shivanna had mentioned that the value of $\mathrm{F}_{\mathrm{ic}}$ depends on the accessibility of the taxa for the treatment of various disease in the study area. Muscle/Bone problem with 81 UR have the highest $F_{\text {ic }}$ value of 0.962 followed by gastro-intestinal disease (GID) with 940 UR and skin care (SC) with 259 UR (Table 5). The lowest $F_{\text {ic }}$ value in the present study was the General Health (GH) category (Cold, fever, immunity boost) with 0.887 which was still

more than the previous maximum $\mathrm{F}_{\mathrm{ic}}$ value report in Shimoga district, Karnataka, India i.e 0.77 in Liver complaints [235]. On the conflict of these report, our study indicated that there was high consistency of the indigenous informant knowledge in the practices of ethnomedicines and utilised the same plants to treat it.

\section{Conclusions}

The present study concluded that the native people in the study area have their unique way of utilizing medicinal plants to treat different kinds of ailments. We documented 93 valuable medicinal plants belonging to 55 families in which Euphorbiaceae and Asteraceae family were the most widely used in the area. Among the plants part, leaves were the most commonly used. No new medicinal taxa were reported but this study is a first quantitative report of ethnomedicine in this region and no informant had reported an adverse effect of herbal medicines. Their traditional pieces of knowledge had been passed on from their elders mostly through word of mouth. This study also revealed that younger generations between the age of 18-30 have little to no knowledge of preparation of herbal medicines and their use as compared to the older age groups. This is mostly due to the availability of modern clinical drugs in the villages. Therefore, the traditional knowledge and practices of medicinal plants in the study area are somehow at risk of dying. This is why it is important to document the valuable knowledge as well as for conservation of the taxa.

The plants which have high usage report and frequency of citation were known to possess numerous phytochemical compounds. The calculated informant consensus factor was extremely high, which means that the acquired data can be used as reference and reliable for ethnopharmacological study in the future. Even though the remedial value of many high cited plants has already been verified, there are still some plants that need to be validated. Hence, they are strongly recommended for further studies to develop alternative drugs.

\section{Abbreviations}

H-Herbs; Sh-Shurbs; Cr-Creeper; T-Tree; UR-Used reports; FC-Frequency of citation; UV-Use value; Lf-Leaf; Br-Bark; Fr-Fruit, Rh-Rhizome; St-Stem; S-Seed; WPWhole Plants; YS-Young Shoot

\section{Declarations}

\section{Ethics approval and consent to participate}

Not applicable. 


\section{Consent for publication}

Not applicable.

\section{Availability of data and materials}

All data generated or analyzed during this study are included in this published article.

\section{Competing interests}

The authors declare that they have no competing interests

\section{Funding}

This research did not receive any specific grant from funding agencies in the public, commercial, or not-for-profit sectors.

\section{Authors Contribution}

Carry out ethnobotanical survey, write the manuscript and analyse the data: TBCL and AK; Study proposer, design the questionnaire and revise the manuscript: TBCL, NMT, RZ, LB and AK; Design the graphical abstract: NMT and AP; Proof reader: RZ and AP.

\section{Acknowledgements}

The authors were thankful to the department of Horticulture, Aromatic and Medicinal Plant, Mizoram University for providing the necessary facilities to complete our work. We also offer our heartfelt gratitude to all the local people of Champhai District, Mizoram who shared their valuable knowledge and precious time for this research.

\section{Authors' information}

${ }^{1}$ Department of Horticulture, Medicinal and Aromatic Plants, School of Earth Science and Natural Resourcess, Mizoram University, 796004 , India; ${ }^{2}$ Department of Botany, School of Life Science, Mizoram University, 796004, India; ${ }^{3}$ Bioprospection and Product Development, CSIR-Central Institute of Medicinal and Aromatic Plants, CIMAP, Lucknow-226015, India

\section{References}

1. Ayyanara M, Ignacimuthub S. Ethnobotanical survey of medicinal plants commonly used by Kani tribals in Tirunelveli hills of Western Ghats, India. $J$ Ethnopharmacol. 2011;134:851-64. https://doi.org/10.1016/j.jep.2011.01.029.

2. Ghimire BK, Tamang JP, Yu CY, Jung SJ, Chung IM. Antioxidant, antimicrobial activity and inhibition of a-glucosidase activity by Betula alnoides Buch. bark extract and their relationship with polyphenolic compounds concentration. Immunopharmacol Immunotoxicol. 2012;34:824-31. https://doi.org/10.3109/08923973.2012.661739

3. Simbo DJ. An ethnobotanical survey of medicinal plants in Babungo, Northwest Region, Cameroon. J Ethnobiol Ethnomed. $2010 ; 6: 8$. https://doi.org/10.1186/1746-4269-6-8

4. Fabricant DS, Farnsworth NR. The value of plants used in traditional medicine for drug discovery. Environ Health Perspect. 2001;109:69-75. https://doi.org/10.1289/ehp.01109s169

5. Kadhirvel K, Ramya S, Sudha TS, Ravi AV, Rajasekaran C, Selvi RV, Jayakumararaj R. Ethnomedicinal survey on plants used by tribals in Chitteri Hills. Environ We Int J Sci Tech. 2010;5:35-46.

6. Rai PK, Lalramnghinglova H. Ethnomedicinal plant resources of Mizoram, India: Implication of traditional knowledge in health care system. Ethnobot Leafl. 2010;3:6.

7. Tikkanen R, Osborn R, Mossialos E, Djordjevic A, Wharton GA. International Health Care System Profiles, India. The Commonwealth Fund. 2020 https://www.commonwealthfund.org/international-health-policy-enter/countries/india Accessed 21.06.2021.

8. Lalramnghinglova H, Jha LK. Ethnomedicine from Mizoram - North East India. Ethnobotany. 1997; 9:105-11.

9. Lalnundanga, Sahoo UK, Jha LK. Ethnobotanical flora in the humid sub-tropical semi-evergreen forest of Mizoram. Proceedings National Conference on Healthcare and Developments of Herbal Medicines, Raipur. 1997.

10. Sharma HK, Chhangte L, Dolui AK. Traditional medicinal plants in Mizoram, India. Fitoterapia. 2001;72(2):146-161. https://doi.org/10.1016/S0367326X(00)00278-1

11. Lalramnghinglova H. Ethno-Medicinal Plants of Mizoram. Bishen Singh Mahendra, Pal Singh, Dehradun, India; 2003.

12. Singh NP, Singh KP, Singh DK. Flora of Mizoram. Botanical Survey of India. Ministry of Environment and Forest. Government of India, Kolkata; 2002

13. Sawmliana M. The Book of Mizoram Plants. Zakhuma P, Aizawl, Mizoram; 2003.

14. Champion GH, Seth SK. A revised survey of the forest types of India. Natraj Publishers, Dehradun; 1968.

15. Champhai district. Government of Mizoram, India. 2021. https://champhai.nic.in/about-district/. Accessed 03 Jan 2021.

16. Zou, TJC: Title of preprint. http://mzuir.inflibnet.ac.in/bitstream/123456789/288/1/T.\%20John\%20Chinliankap\%20Zou\%20(Geography)\%20\%202013.pdf (2013). Accessed 04 Jan 2021. 
17. Khajuria AK, Manhas RK, Kumar H, Bisht NS. Ethnobotanical Study of Traditionally used Medicinal Plants of Pauri District of Uttarakhand, India. J Ethnopharmacol. 2021; 276:114204. https://doi.org/10.1016/j.jep.2021.114204

18. Gazzaneo LRS, Lucena RFP, Albuquerque UP. Knowledge and use of medicinal plants by local specialists in a region of Atlantic Forest in the state of Pernambuco. J Ethnobiol Ethnomedicine. 2005;1:9. https://doi.org/10.1186/1746-4269-1-9

19. Heinrich M, Ankli A, Frei B, Weimann C, Sticher O. Medicinal plants in Mexico: healers' consensus and cultural importance. Soc Sci Med. 1998;47:185971. https://doi.org/10.1016/S0277-9536(98)00181-6

20. Kundan SB, Anupam S. Phytoconstituents and Therapeutic Potential of Allium cepa Linn- A Review. Phcog Rev. 2009;3(5):170-80

21. Kumar KPS, Bhowmik D, Biswaji C, Tiwari P. Allium cepa: A traditional medicinal herb and its health benefits. J Chem Pharm Res. 2010;2(1):283-91.

22. Sharquie KE, Al-Obaidi HK. Onion Juice (Allium cepa L.), A New Topical Treatment for Alopecia Areata. J Dermatol. 2002;29:343-6. https://doi.org/10.1111/j.1346-8138.2002.tb00277.x

23. Teshika JD, Zakariyyah AM, Zaynab T, Zengin G, Rengasamy KR, Pandian SK, Fawzi MM. Traditional and modern uses of onion bulb (Allium cepa L.): A systematic review. Crit. Rev. Food Sci Nutr. 2019;59(1):39-70. https://doi.org/10.1080/10408398.2018.1499074

24. Batiha GES, Beshbishy AM, Elewa Y, Taha A. Chemical Constituents and Pharmacological Activities of Garlic (Allium sativum L.): A Review. Nutrients. 2020; 12:872. http://dx.doi.org/10.3390/nu12030872

25. Miron T, Rabinkov A, Mirelman D, Wilchek M, Weiner L. The mode of action of allicin: Its ready permeability through phospholipid membranes may contribute to its biological activity. Biochim Biophys Acta. 2000;1463:20-30. DOI: 10.1016/S0005-2736(99)00174-1

26. Harris JC, Cottrell SL, Plummer S, Lloyd D. Antimicrobial properties of Allium sativum (garlic). Appl Microbial Biotechnol. 2001;57:282-6. https://doi.org/10.1007/s002530100722

27. Ishtiaq M, Hanif W, Khan MA, Ashraf M, Butt AM. An Ethnomedicinal Survey and Documentation of Important Medicinal Folklore Food Phytonims of Flora of Samahni Valley, (Azad Kashmir) Pakistan. Pakistan J Biol Sci. 2007:10:2241-56. https://doi.org/10.3923/pjbs.2007.2241.2256

28. Singh VK, Singh DK. Pharmacological Effects of Garlic (Allium sativum L.). Annu Rev Biomed Sci. 2008;10:6-26.

29. Arunkumar S, Muthuselvam M. Analysis of Phytochemical Constituents and Antimicrobial Activities of Aloe vera L. Against Clinical Pathogens. World J Agric Sci. 2009;5:572-6.

30. Christaki E, Florou-Paneri PC. Aloe vera: A plant for many uses. J Food Agric Environ. 2010;8:245-9.

31. Sahu PK, Giri DD, Singh R, Pandey P, Gupta S, Shrivastava AK, Kumar A, Pandey KD. Therapeutic and Medicinal Uses of Aloe vera: A Review. J Pharm Pharmacol. 2013;4:599-610. http://dx.doi.org/10.4236/pp.2013.48086

32. Surjushe A, Vasani R, Saple DG. Aloe vera: A short review. Indian Dermatol. 2008;53:163-6. https://dx.doi.org/10.4103\%2F0019-5154.44785

33. Kalaria P, Gheewala P, Chakraborty M, Kamath J. A phytopharmacological review of Alstonia scholaris: A panoramic herbal medicine. Int J Res Ayurveda Pharm. 2012; 3:367-71.

34. Arulmozhi S, Mazumder PM, Ashok P, Narayanan LS. Pharmacological Activities of Alstonia scholaris Linn. (Apocynaceae) - A Review. Pharmacogn Rev. 2007;1:163-70.

35. Baliga MS. Review of the phytochemical, pharmacological and toxicological properties of Alstonia scholaris Linn. R. Br (Saptaparna). Chin J Integr Med. 2012;doi: 10.1007/s11655-011-0947-0

36. Huang XJ, Chen WH, Ji MH, Guo FY, Shu HM, Zheng CJ. Chemical constituents from leaves of Ananas comosus and their biological activities. Zhong Cao Yao. 2015; 46:949-54. doi: 10.7501/j.issn.0253-2670.2015.07.002.

37. Pavan R, Jain S, Kumar A. Properties and therapeutic application of bromelain: A review. Biotechnol Res Int. 2012;6:976203. https://doi.org/10.1155/2012/976203

38. Rathnavelu V, Alitheen NB, Sohila S, Kanagesan S, Ramesh R. Potential role of bromelain in clinical and therapeutic applications. Biomed Rep. 2016;3:283-8. https://doi.org/10.3892/br.2016.720

39. Lin TC, Tanaka T, Nonaka G, Nishioka I, Young TJ. Isolation and Characterization of novel Complex Tannins (Flavano-ellagitannins). Anogeissinin and Anogeissusin-B, from Anogeissus acuminata (Roxb ex Dc) Guill et Perr var Lanccolata Wall ex Clarke. Chem Pharm Bull. 1991;39:1144-7. https://doi.org/10.1248/cpb.39.1144

40. Hemamalini K, Ramu A, Mallu G, Srividya VV, Sravani V, Deepak P, Reddy UV. Evaluation of wound healing activity of different crude extracts of Anogeissus acuminata and Gymnosporia emerginata. Rasayan J Chem. 2011;4:466-71.

41. Monali PM, Padhy R. Antibacterial activity of green silver nanoparticles synthesized from Anogeissus acuminata against multidrug resistant urinary tract infecting bacteria in vitro and host-toxicity testing. J Appl Biomed. 2018;16:120-5. https://doi.org/10.1016/j.jab.2017.11.003

42. Panda SK, Laxmipriya P, Pieter L, Maoxuan L, Johan N, Walter L. Antimicrobial, Anthelmintic, and Antiviral Activity of Plants Traditionally Used for Treating Infectious Disease in the Similipal Biosphere Reserve, Odisha, India Front Pharmacol. 2017;8:658. https://doi.org/10.3389/fphar.2017.00658

43. Panda SS, Girgis AS, Prakash A, Khanna L, Khanna P, Shalaby EM, Fawzy NG, Jain SC. Protective effects of Aporosa octandra bark extract against Dgalactose induced cognitive impairment and oxidative stress in mice. Heliyon. 2018; doi.org/10.1016/j.heliyon.2018.e00951

44. Sahu BP, Gouda P, Patnaik C. Aporosa octandra, A Less Studied Plant Species with Potential Drug Activities-I: Identification of a New Compound from Aqueous Ethanolic Extract of its Stem Bark. Int J Adv Res Sci Eng Tech. 2016;3:2219-24.

45. Vabeiryureilai M, Lalrinzuali K, Rosangkima G, Jagetia GC. Qualitative Phytochemical Analysis and Antioxidant Activity of Aporosa octandra (Buch-Ham. ex D. Don) Extracts. Int J Pharmacol Res. 2014;6:68-73. 
46. Siddiqui BS, Ali ST, Rasheed M, Kardar MN. Chemical Constituents of the Flowers of Azadirachta indica. Helv. Chim Acta. 2003;86:2787-96. https://doi.org/10.1002/hlca.200390229

47. Biswas K, Chattopadhyay I, Banerjee RK, Bandyopadhyay U. Biological activities and medicinal properties of neem (Azadirachta indica). Curr Sci. 2002;82:1336-45.

48. Doshi GM, Nalawade VV, Mukadam AS, Chaskar PK, Zine SP, Somani RR, Une HD. Structural elucidation of chemical constituents from Benincasa hispida seeds and Carissa congesta roots by gas chromatography: Mass spectroscopy. Pharmacogn Res. 2015;7(3):282-93.

https://dx.doi.org/10.4103\%2F0974-8490.157179

49. Al-Snafi AE. The Pharmacological Importance of Benincasa hispida. A review. Int J Pharm Sci Res. 2013;4:165-70.

50. Qadrie ZL, Hawisa NT, Khan MA, Samuel M, Anandan R. Antinociceptive and anti-pyretic activity of Benincasa hispida (Thunb.) cogn. in wistar albino rats. Pak J Pharm Sci. 2009;22:287-90.

51. El-Hawary SS, Hammouda FM, Tawfik WA, Kassem HA, Abdelshafeek KA, El-Shamy SS. Investigation of some chemical constituents, cytotoxicity and antioxidant activities of Beta vulgaris var. altissima cultivated in Egypt. Rasayan J Chem. 2017;10:1391-401.

http://dx.doi.org/10.7324/RJC.2017.1041936

52. El-Beltagi HS, Mohamed HI, Megahed BHM, Gamal M, Safwat G. Evaluation of some chemical constituents, antioxidant, antibacterial and anticancer activities of Beta vulgaris L. root. Fresenius Environ Bull. 2018; 27:6369-78.

53. Maraie NK, A-Jalil TZ, Alhamdany AT, Janab HA. Phytochemical study of the Iraqi Beta vulgaris leaves and its clinical applications for the treatment of different dermatological diseases. World J Pharm Pharm Sci. 2014;3:5-19.

54. Ninfali P, Angelino D. Nutritional and functional potential of Beta vulgaris cicla and rubra. Fitoterapia. 2013;89:188-99. https://doi.org/10.1016/j.fitote.2013.06.004

55. Dũng NX, Mõi LD, Leclercq PA. Constituents of the Bark Oil of Betula alnoides Ham ex. D. Don from Vietnam. J Essent Oil Res. 1995;7:565-6. D0I: 10.1080/10412905.1995.9698589

56. Thu NB, Trung TN, Ha DT, Khoi NM, Hung TV, Hien TT, Yim NH, Bae KH. Screening of Vietnamese Medicinal Plants for Cytotoxic Activity. Nat Prod Sci. 2010;16:43-9.

57. Yang DS, Yang YP, Yong-hong Y, Xiao-li L. Chemical Constituents of Bischofia javanica. Nat Prod Res Dev. 2013;25:1056-9. DOI: 10.3969/j.issn.10016880.2013.08.009.

58. Khan MR, Kihara M, Domoloso A. Anti-microbial activity of Bidens pilosa, Bischofia javanica, Elmerillia papuana and Sigesbekia orientalis. Fitoterapia. 2001; 72:662-5. https://doi.org/10.1016/S0367-326X(01)00261-1

59. Lingadurai S, Roy S, Joseph RV, Nath LK. Antileukemic activity of the leaf extract of Bischofia javanica blume on human leukemic cell lines. Indian J Pharmacol. 2011;43:143-9. https://dx.doi.org/10.4103\%2F0253-7613.77348

60. Anadebe VC, Okafor NA, Ezeugo JO, Amanjide IJ, Ogide BC. GC-MS Analysis of Phytochemical Compounds in Cajanus cajan Leaf. J Chem Pharm Res. 2017;9:360-3.

61. Pal D, Mishra P, Sachan N, Ghosh AK. Biological activities and medicinal properties of Cajanus cajan (L) Millsp. J Adv Pharm Technol Res. 2011;2:207-14. https://dx.doi.org/10.4103\%2F2231-4040.90874

62. Tu Y, Sun L, Guo M, Chen W. The medicinal uses of Callicarpa L. in traditional Chinese medicine: An ethnopharmacological, phytochemical and pharmacological review. J Ethnopharmacol. 2013;146:465-81. https://doi.org/10.1016/j.jep.2012.12.051

63. Zhu HB, Li BM, Liu C, Chen RY. Chemical constituents of Camellia sinensis var. assamica. Zhongguo Zhong yao za. 2013;38:1386-89. DOI: $10.4268 / \mathrm{cjcmm} 20130925$.

64. Sarangi AB. Medicinal and therapeutic potentialities of tea (Camellia sinensis L.) - A review. Food Res Int. 2009;42:529-35. https://doi.org/10.1016/j.foodres.2009.01.007

65. Hamilton-Miller JM. Antimicrobial properties of tea (Camellia sinensis L.) Antimicrobe. Agents Chemother. 1995;39:2375-7. https://dx.doi.org/10.1128\%2Faac.39.11.2375

66. Grutenherman F, Russo E. Cannabis and Cannabinoids, Pharmacology, Toxicology and Therapeutic Potential. The Howarth Integrative Healing Press, New York. 2002.

67. Nuutinen T. Medicinal properties of terpenes found in Cannabis sativa and Humulus lupulus. Eur J Med Chem. 2018;157:198-228. https://doi.org/10.1016/j.ejmech.2018.07.076

68. Siddiqui BS, Aslam H, Ali ST, Khan S, Begum S. Chemical constituents of Centella asiatica. J Asian Nat Prod Res. 2007;9:407-14. DOI: 10.1080/10286020600782454

69. Kalshetty P, Aswar U, Bodhankar S, Sinnathambi A, Mohan V, Thakurdesai P. Antidepressant effects of standardized extract of Centella asiatica L in olfactory bulbectomy model. Biomed Aging Pathol. 2012;2:48-53. https://doi.org/10.1016/j.biomag.2012.03.005

70. Nugrahaningsih DAA, Purnomo E. Protective Effect of Centella asiatica (L.) Urban on Heart and Kidney of Rat with Subtotal Nephrectomy. Der Pharma Lett. 2018;10:21-9

71. Punturee K, Wild CP, Kasinrerk W, Vinitketkumnuen U. Immunomodulatory Activities of Centella asiatica and Rhinacanthus nasutus Extracts. Asian Pac J Cancer Prev. 2005;6:396-400.

72. Marin A, Ferreres F, Tomás-Barberán FA, Gil MI. Characterization and Quantitation of Antioxidant Constituents of Sweet Pepper (Capsicum annuum L.). J Agric Food Chem. 2004;52:3861-9. https://doi.org/10.1021/jf0497915

Page $21 / 30$ 
73. Khan FA, Mahmood T, Ali M, Saeed A, Maalik A. Pharmacological importance of an ethnobotanical plant: Capsicum annuum L. Nat Prod Res. 2014;28:1267-74. https://doi.org/10.1080/14786419.2014.895723

74. Yogiraj V, Goyal PK, Chauhan CS, Goyal A, Vyas B. Carica papaya Linn: An Overview. Int J Herbal Med. 2014;2:1-8.

75. Adam Y, Nasaruddin AA, Zuraini A, Arifah AK, Zakaria MOFZ, Somchit MN. Diuretic Activity of Roots from Carica papaya L. and Ananas comosus L. Int J Pharm Sci Rev Res. 2013;23:163-7.

76. Krishna KL, Paridhavi M, Patel KL. Review on nutritional, medicinal and pharmacological properties of Papaya (Carica papaya Linn.). Nat Prod Radiance. 2008;7:364-73. http://nopr.niscair.res.in/handle/123456789/5695

77. Prakash D, Upadhyay G, Gupta C, Pushpangadan P, Singh KK. Antioxidant and free radical scavenging activities of some promising wild edible fruits. Int Food Res J. 2012;19:1109-16.

78. Ferreres F, Pereira DM, Valentão P, Andrade PB, Seabra RM, Sottomayor M. New Phenolic Compounds and Antioxidant Potential of Catharanthus roseus. J Agric Food Chem. 2008;56:9967-74.

79. Aslam J, Khan SH, Siddiqui ZH, Fatima Z, Maqsood M, Bhat MA, Nasim SA, Ilah A, Ahmad IZ, Khan SA, Mujib A. Catharanthus roseus (L.) G. Don. An important drug: it's applications and production. Pharmacie Globale. 2010;4:1-16.

80. Gajalakshmi S, Vijayalakshmi SD, Rajeswari V. Pharmacological activities of Catharanthus roseus: A perspective review. Int J Pharm Bio Sci. 2013;4:4319.

81. Hassan HM, Eldesoky AM, Al-Rashdi A, Ahmed HM. Green Corrosion Electrochemistry: Cheilocostus speciosus Extract (CSE) Investigated Electroanalytically as a Potential Green Corrosion Inhibitor for Copper within Acidic Solution. Int J Emerg Trends Eng Develop. 2016;7:72-98.

82. Ayam VS, Gogoi P. Evaluation of Antioxidant Activity of the Selected frequently used Traditional Wild Medicinal Plants from Lakhimpur, Assam (India). Pleione. 2018;12:187-92. Doi:10.26679/Pleione.12.2.2018.

83. Benelli G, Govindarajan M, Rajeswary M, Vaseeharan B, Alyahya SA, Alharbi NS, Kadaikunnan S, Khaled JM, Maggi F. Insecticidal activity of camphene, zerumbone and a-humulene from Cheilocostus speciosus rhizome essential oil against the Old-World bollworm, Helicoverpa armigera. Ecotoxicol Environ Saf. 2018;148:781-6. https://doi.org/10.1016/j.ecoenv.2017.11.044

84. Owolabi MS, Ogundajo A, Yusuf KO, Lajide L, Villanueva HE, Tuten JA, Setzer WN. Chemical Composition and Bioactivity of the Essential Oil of Chromolaena odorata from Nigeria. Rec Nat Prod. 2010;4:72-8.

85. Taiwo OB, Olajide OA, Soyannwo 00, Makinde JM. Anti-Inflammatory, Antipyretic and Antispasmodic Properties of Chromolaena Odorata. Pharm Biol. 2000;38:367-70. https://doi.org/10.1076/phbi.38.5.367.5970

86. Vaisakh MN, Pandey A. The invasive weed with healing properties: A review on Chromolaena odorata. Int J Pharm Sci Res. 2012;3:80-3.

87. Abyaneh M, Shams-Ghahfarokhi M, Rezaee MB, Jaimand K, Alinezhad S, Saberi R, Yoshinari T. Chemical composition and antiaflatoxigenic activity of Carum carvi L., Thymus vulgaris and Citrus aurantifolia essential oils. Food Control, 2009;20:1018-24. https://doi.org/10.1016/j.foodcont.2008.12.007

88. Pathan RK, Gali PR, Pathan P, Gowtham T, Pasupuleti S. In vitro Antimicrobial Activity of Citrus aurantifolia and its phytochemical screening. Asian Pac J Trop Dis. 2012;2:328-31. https://doi.org/10.1016/S2222-1808(12)60176-5

89. Taur DJ, Kulkarni VB, Patil RY, Patil RN. Anthelmintic activity of Ocimum sanctum and Citrus aurantifolia oils. Pharmacologyonline. 2009;3:495-9.

90. Xu YR, Zhang KF, Xie QJ, Lin JX, Huan KX, Liao Y. Chemical Constituents from Young Fruits of Citrus maxima cv. Shatian. Zhong yao cai. 2015;38:187981.

91. Abirami A, Nagarani G, Siddhuraju P. Antimicrobial activity of crude extract of Citrus hystrix and Citrus maxima. Int J Pharm Sci. 2013;4:1-5.

92. Shivananda A, Rao DM, Jayaveera KN. Analgesic and anti-inflammatory activities of Citrus maxima (J. Burm) Merr in animal models. Res J Pharm Biol Chem Sci. 2013;4:1800-10. http://rjpbcs.com/pdf/2013_4(2)/

93. Sudeep DS, Karunakar H. Antiobesity activity of ethanolic extract of Citrus maxima leaves on cafeteria diet induced and drug induced obese rats. Res $\mathrm{J}$ Pharm Tech. 9, 907-912. http://dx.doi.org/10.5958/0974-360X.2016.00173.6

94. Kefford, J.F., 1960. The Chemical Constituents of Citrus Fruits. Adv Food Res. 2016;9:285-372. https://doi.org/10.1016/S0065-2628(08)60278-5

95. Szczykutowicz MK, Szopa A, Ekiert H. Citrus limon (Lemon) Phenomenon-A Review of the Chemistry, Pharmacological Properties, Applications in the Modern Pharmaceutical, Food, and Cosmetics Industries, and Biotechnological Studies. Plants. 2020;9:119. https://doi.org/10.3390/plants9010119

96. Jadeja RN, Thounaojam MC, Singh TB, Devkar RV, Ramachandran AV. Traditional uses, phytochemistry and pharmacology of Clerodendron glandulosum Coleb - A review. Asian Pac J Trop Med. 2012;5:1-6. https://doi.org/10.1016/S1995-7645(11)60236-8

97. Prajapati R, Kalariya M, Umbarkar R, Parmar S, Sheth N. Colocasia esculenta: A potent indigenous plant. Int J Nutr Pharmacol Neurol Dis. 2011;1:90-6. http://www.ijnpnd.com/text.asp?2011/1/2/90/84188

98. Agyare C, Boakye YD, Apenteng JA, Dapaah SO, Appiah T, Adow A. Antimicrobial and Anti-Inflammatory Properties of Anchomanes difformis (BI.) Engl. and Colocasia esculenta (L.) Schott. Biochem Pharmacol. 2016;5:1. DOI: 10.4172/2167-0501.1000201

99. Kubde MS, Khadabadi SS, Farooqui IA, Deore SL. In-vitro anthelmintic activity of Colocasia esculenta. Der Pharma Lett. 2010;2:82-5.

100. Patil BR, Ageely HM. Antihepatotoxic activity of Colocasia esculenta leaf juice. Int J Adv Biotech Res. 2011;2:296-304.

101. Koné WM, Vargas M, Keiser J. Anthelmintic activity of medicinal plants used in Côte d'Ivoire for treating parasitic diseases. Parasitol Res. 2012;110:235162. https://doi.org/10.1007/s00436-011-2771-z

102. Joshi RK. Study on essential oil composition of the roots of Crassocephalum crepidioides (Benth.) S. Moore. J Chil Chem Soc. 2014;59:2363-65. http://dx.doi.org/10.4067/S0717-97072014000100025

Page 22/30 
103. Tomimori K, Nakama S, Kimura R, Tamaki K, Ishikawa C, Mori N. Antitumor activity and macrophage nitric oxide producing action of medicinal herb, Crassocephalum crepidioides. BMC Complement Altern Med. 2012;12:1-11. https://doi.org/10.1186/1472-6882-12-78

104. Bahar E, Akter KM, Lee GH, Lee HY, Rashid HO, Choi MK, Bhattarai KR, Hossain MMM, Ara J, Mazumder K, Raihan O. $\beta$-Cell protection and antidiabetic activities of Crassocephalum crepidioides (Asteraceae) Benth. S. Moore extract against alloxan-induced oxidative stress via regulation of apoptosis and reactive oxygen species (ROS). BMC Complement Altern Med. 2017;17:179. https://doi.org/10.1186/s12906-017-1697-0.

105. Rezig L, Chouaibi M, Msaada K, Hamdi H. Chemical composition and profile characterisation of pumpkin (Cucurbita maxima) seed oil. Ind Crops Prod. 2012;37: 82-7. https://doi.org/10.1016/j.indcrop.2011.12.004

106. Md PM, Md TK. Overview on Cucurbita maxima seed. J Dent Med Sci. 2017;16:29-33. DOI: 10.9790/0853-1603132933

107. Borah A. Chemical composition, antioxidant, anti-inflammatory, anti-microbial and in-vitro cytotoxic efficacy of essential oil of Curcuma caesia Roxb. leaves: An endangered medicinal plant of North East India. Ind Crops Prod. 2019;129:448-54. https://doi.org/10.1016/j.indcrop.2018.12.035

108. Baghel SS, Baghel RS, Sharma K, Sikarwar I. Pharmacological activities of Curcuma caesia. Int J Green Pharm. 2013;7:1-5. http://dx.doi.org/10.22377/ijgp.v7i1.287

109. Lateef EA. Bioactive chemical constituents of Curcuma longa L. rhizomes extract inhibit the growth of human hepatoma cell line (HepG2). Acta Pharm. 2016; 66:387-98. https://doi.org/10.1515/acph-2016-0028

110. Krup V, Prakash LH, Harini A. Pharmacological Activities of Turmeric (Curcuma longa Linn): A Review. J Homeop Ayurv Med. $2013 ; 2: 133$. http://dx.doi.org/10.4172/2167-1206.1000133

111. Wu XY, Chao ZM, Wang C, Tan ZG, Sun W. Chemical constituents contained in fatty oil from seeds of Cucumis sativus. Zhongguo Zhong Yao Za Zhi. 2012;37:3252-5.

112. Saeedi R, Sultana A, Rahman K. Ethnomedicinal uses and pharmacological activities of different parts of Cucumis sativus Linn: An update. Int J Pharm. Sci Res. 2020;11:1549-56. Doi: 10.13040/IJPSR.0975-8232.11, 4, 1549-56.

113. Zhu SH, Zhang QJ, Chen Q, Zhou T, Yao RJ. Study on the chemical constituents of Dichrocephala integrifolia. Zhong yao cai. 2010;33:53-5.

114. Emégam NK, Nguepi MSD, Lambou AF, Okomolo FCM, Sotoing GT, Bougolla DP, Pale S, Kameni JSN, Bum EN. Antioxidant properties of Dichrocephala integrifolia (Asteraceae) in a mouse model of monosodium glutamate-induced neurotoxicity. Afr J Tradit Complement Altern Med. 2017;14:147-55.

115. Kouémou NE, Taiwe GS, Moto FC, Pale S, Ngoupaye GT, Njapdounke JS, Nkantchoua GC, Pahaye DB, Bum EN. Nootropic and neuroprotective effects of Dichrocephala integrifolia on scopolamine mouse model of Alheimer's disease. Front Pharmacol. 2017. https://doi.org/10.3389/fphar.2017.00847

116. Lee CL, Yen MH, Hwang TL, Yang JC, Peng CY, Chen CJ, Chang WY, Wu YC. Anti-inflammatory and cytotoxic component from Dichrocephala integrifolia. Phytochem Lett. 2015;12:237-42. https://doi.org/10.1016/j.phytol.2015.04.012

117. Rene K, Olivier FG, Jacqueline NT, Hortense GK, Christine FM. Antimicrobial and antidiarrheal effects of four Cameroon medicinal plants: Dichrocephala integrifolia, Dioscorea preusii, Melenis minutiflora, and Tricalysia okelensis. Int J Curr Pharma Res. 2015;7:21-4.

118. Gandhi D, Mehta P. Dillenia indica Linn. and Dillenia pentagyna Roxb.: Pharmacognostic, Phytochemical and Therapeutic aspects. J App Pharm Sci. 2013;3:134-42. DOI: 10.7324/JAPS.2013.31124

119. Cheng WY, Kuo YH, Huang CJ. Isolation and Identification of Novel Estrogenic Compounds in Yam Tuber (Dioscorea alata Cv. Tainung No. 2). J Agric Food Chem. 2007;55:7350-8. DOI: 10.1021/jf0711690

120. Das A, Chaudhuri D, Chatterjee A. Study of antioxidant and reactive oxygen species scavenging activity of the edible tuber of "Greater Yam" (Dioscorea alata L.) from North-east India. Asian J Pharm Clin Res. 2012;5:74-84.

121. Maithili V, Dhanabal SP, Mahendran S, Vadivelan R. Antidiabetic activity of ethanolic extract of tubers of Dioscorea alata in alloxan induced diabetic rats. Indian J Pharmacol. 2011;43:455-9. https://dx.doi.org/10.4103\%2F0253-7613.83121

122. Nono NR, Nzowa KL, Barboni L, Tapondjou AL. Drymaria cordata (Linn.) Willd (Caryophyllaceae): Ethnobotany, Pharmacology and Phytochemistry. Adv Biol Chem. 2014;4:160-7. http://dx.doi.org/10.4236/abc.2014.42020

123. Liu HB, Zhang CR, Dong SH, Yang SP, Sun Q, Geng MY, Yue JM. Sesquiterpenes from Dysoxylum oliganthum and Dysoxylum excelsum. J Asian Nat Prod Res. 2012;14:224-34. DOI: 10.1080/10286020.2011.645810

124. Jayashree R, Albert S, Minaram N. Medicinal plants of North Cachar Hills district of Assam used by the Dimasa tribe. Indian J Tradit Knowl. 2012;11:5207. http://nopr.niscair.res.in/handle/123456789/14395

125. Sharma BK, Ramashanker SG, Rahaman L, Nath N, Kaipeng DL. Plant based folk treatments from North East India for jaundice. (An overview). J Med Plants Stud. 2016.;4:234-47.

126. Lasekan O, Buettner A, Christlbauer M. Investigation of important odorants of palm wine (Elaeis guineensis). Food Chem. 2007;105:15-23. https://doi.org/10.1016/j.foodchem.2006.12.052

127. Owoyele BV, Owolabi GO. Traditional oil palm (Elaeis guineensis Jacq.) and its medicinal uses: A review. Tang Humanitas Medicines. 2014. http://dx.doi.org/10.5667/tang.2014.0004

128. Yin NGS, Abdullah S, Phin CK. Phytochemical constituents from leaves of Elaeis guineensis and their antioxidant and antimicrobial activities. Int J Pharm Pharm Sci. 2013;5:137-40.

129. Shivprasad M, Varsha J. GC-MS Screening of Some Bioactive Compounds from Methanolic Extract of Medicinally Relevant Wild Edible Plant Parts. Int J Sci Res Sci Tech. 2018;4:49-56

130. Sethiya NK, Brahmbhat K, Chauhan B, Mishra SH. Pharmacognostic and phytochemical investigation of Ensete superbum (Roxb.) Cheesman pseudostem. Indian J Nat Prod Resour. 2016;7:51-8. http://nopr.niscair.res.in/handle/123456789/34105

Page 23/30 
131. Akter K, Barnes EC, Loa-Kum-Cheung WL, Yin P, Kichu M, Brophy JJ, Barrow RA, Imchen I, Vemulpad SR, Jamie JF. Antimicrobial and antioxidant activity and chemical characterisation of Erythrina stricta Roxb. (Fabaceae). J Ethnopharmacol. 2016;185:171-81. https://doi.org/10.1016/j.jep.2016.03.011.

132. Araújo-Júnior JX, de Oliveira MS, Aquino PG, Alexandre-Moreira MS, Sant'Ana AE. A phytochemical and ethnopharmacological review of the genus Erythrina in Phytochemicals. In: Rao V, editors. A Global Perspective of Their Role in Nutrition and Health. London: InTech; 2012. p. 327-49.

133. Subhashini N, Purnima S, Devi JA, Thirupathi AT, Lavanya N. Anti-Inflammatory activity of Erythrina stricta Roxb. in Albino Rats. Int J Pharm Tech Res. 2011;3:1014-8.

134. Hada S, Yadav DK, Roat P, Kumari N. Eulophia Nuda: A Review of Its Traditional Uses, Phytochemistry and Pharmacology. Pharm Chem J. 2020;54:40-5. DOI: $10.1007 / \mathrm{s} 11094-020-02152-8$

135. Jain JB, Kumane SC, Bhattacharya S. Medicinal flora of Madhya Pradesh and Chattisgarh - A Review. Indian J Tradit Knowl. 2006;5:237-42. http://nopr.niscair.res.in/handle/123456789/6845

136. Pascal OA, Bertrand AEV, Esaïe T, Sylvie HAM, Eloi AY. A review of the ethnomedical uses, phytochemistry and pharmacology of the Euphorbia genus. J Pharm Innov. 2017;6:34-9

137. Rauf A, Muhammad N, Qaisar M, Uddin G, Hussain I. Preliminary Antinociceptive Studies of Methanol Extract of Euphorbia milli. Middle-East J Med Plants Res. 2012;1:68-70. DOI: 10.5829/idosi.mejmpr.2011.1.3.1115

138. Saleem H, Zengin G, Locatelli M, Mollica A, Ahmad I, Mahomoodally FM, Abidin SAZ, Ahemad N. In vitro biological propensities and chemical profiling of Euphorbia milii Des Moul (Euphorbiaceae): A novel source for bioactive agents. Ind Crops Prod. 2019;130:9-15. https://doi.org/10.1016/j.indcrop.2018.12.062

139. Wang P, Xie C, An L, Yang X, Xi Y, Yuan S, Zhang C, Tuerhong M, Jin DQ, Lee D, Zhang J. Bioactive Diterpenoids from the Stems of Euphorbia royleana. J Nat Prod. 2019;82:183-93. https://doi.org/10.1021/acs.jnatprod.8b00493

140. Ashraf A, Sarfraz RA, Rashid MA, Shahi M. Antioxidant, antimicrobial, antitumor, and cytotoxic activities of an important medicinal plant (Euphorbia royleana) from Pakistan. J Food Drug Anal. 2015;23:109-15. https://doi.org/10.1016/j.jfda.2014.05.007.

141. Bani S, Kaul A, Jaggi BS, Suri KA, Suri OP, Sharma OP. Anti-inflammatory activity of the hydrosoluble fraction of Euphorbia royleana latex. Fitoterapia. 2000;71:655-62. https://doi.org/10.1016/S0367-326X(00)00225-2

142. Wang GC, Liang JP, Wang Y, Li Q, Ye WC. Chemical Constituents from Flueggea virosa. Chinese J Nat Med. 2008;6:251-3. https://doi.org/10.1016/S18755364(09)60022-4

143. Chao CH, Cheng JC, Shen DY, Huang HC, Wu YC, Wu TS. Terpenoids from Flueggea virosa and their anti-hepatitis $\mathrm{C}$ virus activity. Phytochemistry. 2016;128: 60-70. https://doi.org/10.1016/j.phytochem.2016.04.003

144. Ezeonwumelu JOC, Omar AN, Ajayi AM, Okoruwa AG, Tanayen JK, Kiplagat DM, Okpanachi OA, Abba S, Ezekiel I, Onchweri AN, Okonkwo CO. Phytochemical screening, acute toxicity, anti-inflammatory and anti-pyretic studies of aqueous extract of the root of Flueggea virosa (Roxb. ex Willd.) in rats. Int J Pharm Biomed Sci. 2012;3:128-35.

145. Wang GC, Li T, Deng FY, Li YL, Ye WC. Five new phenolic glycosides from Hedyotis scandens. Bioorganic Med Chem Lett. 2013;5:1379-82. https://doi.org/10.1016/j.bmcl.2012.12.077

146. Rahman MA, Uddin SB, Wilcock CC. Medicinal plants used by Chakma tribe in Hill Tracts districts of Bangladesh. Indian J Tradit Knowl. 2007;6:508-17. http://nopr.niscair.res.in/handle/123456789/991

147. Subba B, Basne P. Antimicrobial activity of some medicinal plants from east and central part of Nepal. Int J Appl Sci Biotechnol. 2014;2:88-92. https://doi.org/10.3126/ijasbt.v2i1.9697

148. Jadhav VM, Thorat RM, Kadam VJ, Sathe NS. Hibiscus rosa sinensis Linn - "Rudrapuspa”: A Review. J Pharm Res. 2009;2:1168-73.

149. Mak YM, Chuah LO, Ahmad R, Bhat R. Antioxidant and antibacterial activities of hibiscus (Hibiscus rosa-sinensis L.) and Cassia (Senna bicapsularis L.) flower extracts. J King Saud Univ Sci. 2013;25(4):275-82. https://doi.org/10.1016/j.jksus.2012.12.003

150. Fu J, Dai L, Lin Z, Lu H. Houttuynia cordata Thunb: A Review of Phytochemistry and Pharmacology and Quality Control. Chinese Med. 2013;4:101-23. Doi: 10.4236/cm.2013.43015.

151. Mohanraj R, Sivasankar S. Sweet Potato (Ipomoea batatas [L.] Lam) - A Valuable Medicinal Food: A Review. J Med Food. 2014;17:733-41. https://doi.org/10.1089/jmf.2013.2818

152. Hossain S, Ahmed R, Bhowmick S, Al Mamun A, Hashimoto M. Proximate composition and fatty acid analysis of Lablab purpureus (L.) legume seed: implicates to both protein and essential fatty acid supplementation. Springer Plus. 2016;5:1899. https://doi.org/10.1186/s40064-016-3587-1

153. Al-Snafi AE. The pharmacology and medical importance of Dolichos lablab (Lablab purpureus)-A review. IOSR J Pharm. 2017;7:22-30.

154. Wei JC, Wang PC, Zhou XI. The caffeoyl phenylethanoid glycosides from Lindernia ruellioides and their anti-HBV effects. J Asian Nat Prod Res. 2018;20: 757-62. https://doi.org/10.1080/10286020.2017.1357549

155. Das AK, Nongmaithem R. Phytochemical study of selected medicinal plants used by the maring tribe of Chandel district, Manipur, India. J Pharmacogn Phytochem. 2019;8:2155-60.

156. Kuruvilla GR, Neeraja M, Srikrishna A, Rao SGRS. A new quinone from Maesa indica (Roxb) A DC, (Myrsinaceae). Indian J Chem Sect B. $2010 ; 49: 1637-41$.

157. Patil A, Jadhav V, Arvindekar A, More T. Antidiabetic activity of Maesa indica (Roxb). stem bark in Streptozotoc in induced diabetic rats. American J Phytomed Clin Ther. 2014;2:957-62.

158. Wei L, Wee W, Siong J, Syamsumir D. Characterization of antimicrobial, antioxidant, anticancer property and chemical composition of Michelia champaca seed and flower extracts. Stamford J Pharm Sci. 2011;4:19-24. https://doi.org/10.3329/sjps.v4i1.8862 
159. Vimala R, Nagarajan S, Alam M, Susan T, Joy S. Anti-inflammatory and Antipyretic Activity of Michelia champaca Linn., (White Variety), Ixora Brachiata Roxb. and Rhynchosia Cana (Willd.) D.C. Flower Extract. Indian J Exp Biol. 1997; 35;1310-14.

160. Dan GD, Yi Z, Wei LE, Tao W, Min HL. Chemical constituents of Mangifera indica leaves (I). Zhong Cao Yao. 2011;42:428-31. http://www.ceps.com.tw/ec/ecJnllntro.aspx?Jnliid=2790

161. Parvez GMM. Pharmacological activities of mango (Mangifera indica): A review. J Pharmacogn Phytochem 2016;5:1-7.

162. Pandey AK, Rai MK, Acharya D. Chemical Composition and Antimycotic Activity of the Essential Oils of Corn Mint (Mentha arvensis) and Lemon Grass (Cymbopogon flexuosus) Against Human Pathogenic Fungi. Pharm Biol. 2003;41: 421-5. https://doi.org/10.1076/phbi.41.6.421.17825

163. Thawkar BS, Jawarkar AG, Kalamkar PV, Pawar KP, Kale MK. Phytochemical and pharmacological review of Mentha arvensis. Int J Green Pharm. 2016;10:2. http://dx.doi.org/10.22377/ijgp.v10i2.643

164. Chow YL, Quon HH. Chemical constituents of the heartwood of Mesua ferrea. Phytochemistry. 1968;7:1871-4. https://doi.org/10.1016/S00319422(00)86662-5

165. Chahar MK, Sanjaya KDS, Geetha L, Lokesh T, Manohara KP. Mesua ferrea L.: A review of the medical evidence for its phytochemistry and pharmacological actions. Afr J Pharm Pharmacol. 2013;7:211-9. https://doi.org/10.5897/AJPP12.895

166. Shao H, Nan P, Peng S, Zhang C. Study of chemical constituents of essential oil from flowers of Mikania micrantha. Zhong yao cai. 2001;24:341-2.

167. Dev UK, Md Hossain T, Md Islam Z. Phytochemical investigation, antioxidant activity and antihelmintic activity of Mikania micrantha leaves. World J Pharma Res. 2015;4:121-33.

168. Lentz DL, Clark AM, Hufford CD, Meurer-Grimes B, Passreiter CM, Cordero J, Ibrahimi O, Okunade AL. Antimicrobial properties of Honduran medicinal plants. J Ethnopharmacol. 1998;63:253-63. https://doi.org/10.1016/S0378-8741(98)00100-7

169. Yuan K, Lü JL, Yin MW. Chemical constituents of C-glycosylflavones from Mimosa pudica. Yao Xue Xue Bao. 2006;41:435-8.

170. Joseph B, George J, Mohan J. Pharmacology and Traditional Uses of Mimosa pudica. Int J Pharma Sci Drug Res. 2013;5:41-4.

171. Farrag EK, Kassem MES, Bayoumi D, Shaker SE, Afifi MS. Phytochemical study, phenolic profile and antigastric ulcer activity of Morus macroura Miq. fruits extract. J Appl Pharm Sci. 2017;7:152-60. DOI: 10.7324/JAPS.2017.70527

172. Sidhu JS, Zafar TA. Bioactive compounds in banana fruits and their health benefits. Food Qual Saf. 2018;2:183-8. https://doi.org/10.1093/fqsafe/fyy019

173. Jyothirmayi N, Rao NM. Banana Medicinal Uses. J Med Sci Tech. 2015;4:152-60.

174. Mathew NS, Negi PS. Traditional uses, phytochemistry and pharmacology of wild banana (Musa acuminata Colla): A review. J Ethnopharmacol. 2017;196:124-40. https://doi.org/10.1016/j.jep.2016.12.009

175. Kim NC, Desjardins AE, Wu CD, Kinghorn AD. Activity of Triterpenoid Glycosides from the Root Bark of Mussaenda macrophylla against Two Oral Pathogens. J Nat Prod. 1999;62:1379-84. https://doi.org/10.1021/np9901579

176. Chowdhury SR, Akter S, Sharmin T, Islam F, Quadery TM. Antimicrobial activity of five medicinal plants of Bangladesh. J Pharmacogn Phytochem. 2013;2:164-70.

177. Shadia E, El-Aziz A, Omer EA, Sabra AS. Chemical Composition of Ocimum americanum Essential Oil and Its Biological Effects Against, Agrotis ipsilon, (Lepidoptera: Noctuidae). Res J Agric Biol Sci. 2007;3:740-7.

178. Hakkim FL, Arivazhagan G, Boopathy R. Antioxidant property of selected Ocimum species and their secondary metabolite content. J Med Plant Res. 2008;2: 250-7.

179. Thaweboon S, Thaweboon B. In vitro antimicrobial activity of Ocimum americanum L. essential oil against oral microorganisms. Southeast Asian J Trop Med Public Health. 2009;40:1025-33.

180. Ahad A, Ganai AA, Sareer O, Najm MZ, Kausar MA, Mujeeb M, Siddiqui WA. Therapeutic potential of oroxylum indicum: a review. J Pharma Res Opinion. 2012;2:163-72.

181. Singh HV, Chaudhary AK. A Review on the Taxonomy, Ethnobotany, Chemistry and Pharmacology of Oroxylum indicum Vent. Indian J Pharm Sci. 2011;73:483-90. https://dx.doi.org/10.4103\%2F0250-474X.98981

182. Hussain A, Oves M, Alajmi MF, Hussain I, Amir S, Ahmed J, Rehman MT, El-Seedi HR, Ali I. Biogenesis of ZnO nanoparticles using Pandanus odorifer leaf extract: anticancer and antimicrobial activities. RSC Adv. 2019;9:15357-69. https://doi.org/10.1039/C9RA01659G

183. Angami T, Bhagawati R, Touthang L, Makdoh B, Bharati KA, Silambarasan R, Ayyanar M. Traditional uses, phytochemistry and biological activities of Parkia timoriana (DC.) Merr., an underutilized multipurpose tree bean: A review. Genet Resour Crop Evol. 2018.;65:679-92. https://doi.org/10.1007/s10722017-0595-0.

184. Gaire BP, Subedi L. Phytochemistry, pharmacology and medicinal properties of Phyllanthus emblica Linn. Chin J Integr Med. 2014. D0I: 10.1007/s11655014-1984-2

185. Lin HY, Yuan CY, Xin WY, Peng LD, Juan CW, Lei LJ, Lai LF. Chemical constituents of Picria fel-terrae. Guangxi Zhiwu / Guihaia. 2010;30:887-90.

186. Kumarasingha R, Karpe AV, Preston S, Yeo TC, Lim DS, Tu CL, Luu J, Simpson KJ, Shaw JM, Gasser RB, Beale DJ. Metabolic profiling and in vitro assessment of anthelmintic fractions of Picria fel-terrae Lour. Int J Parasitol Drugs Drug Resist. 2016;6:171-8.

https://doi.org/10.1016/j.ijpddr.2016.08.002

187. Satria D, Silalahi J, Haro G, llyas S, Hsb PAZ. Antioxidant and Antiproliferative Activities of an Ethylacetate Fraction of Picria fel-terrae Lour. Herbs. Asian Pac J Cancer Prev. 2017;18;399-403. https://dx.doi.org/10.22034\%2FAPJCP.2017.18.2.399

188. Samuelsen AB. The traditional uses, chemical constituents and biological activities of Plantago majorL. A review. J Ethnopharmacol. 2000;71:1-21. DOI: 10.13040/IJPSR.0975-8232.11(4).1549-56

Page 25/30 
189. Begum S, Hassan SI, Ali SN, Siddiqui BS. Chemical constituents from the leaves of Psidium guajava, Nat Prod Res. 2004;18:135-40. https://doi.org/10.1080/14786410310001608019

190. Martha R, Gutiérrez P, Mitchell S, Solis RS. Psidium guajava: A review of its traditional uses, phytochemistry and pharmacology. J Ethnopharmacol. 2008;117:1-27. https://doi.org/10.1016/j.jep.2008.01.025

191. Tai Z, Zhang F, Cai L, Shi J, Cao Q, Ding Z. Flavonol glycosides of Pseudodrynaria coronans and their antioxidant activity. Chem Nat Compd. 2012; 48:221-4. https://doi.org/10.1007/s10600-012-0209-1

192. Jasuja ND, Saxena R, Chandra S, Sharma R. Pharmacological characterization and beneficial uses of Punica granatum. Asain J Plant Sci. 2012;11:25167.

193. Djakpo O, Yao W. Rhus chinensis and Galla chinensis - Folklore to Modern Evidence: Review. Phytother Res. 2010;24:1739-47. https://doi.org/10.1002/ptr.3215

194. Rishi P, Bimala S. Extraction and Isolation of Chemical Constituents from Schima wallichii Bark. Int J Eng Sci Res Tech. 2014;3:175-80.

195. Das S, Ghosh LK. Evaluation of Analgesic, Antipyretic and Anti-Inflammatory Activity of Different Fractions of Schima wallichii Barks. Pharmacologia. 2013;4:400-3. http://dx.doi.org/10.5567/pharmacologia.2013.400.403

196. Sarbadhikary SB, Bhowmik S, Datta BK, Mandal NC. Antimicrobial and antioxidant activity of leaf extracts of two indigenous angiosperm species of Tripura. Int J Curr Microbiol App Sci. 2015;4:643-55.

197. Wang D, Huang L, Chen S. Senecio scandens Buch-Ham.: A review on its ethnopharmacology, phytochemistry, pharmacology, and toxicity. J Ethnopharmacol. 2013;149:1-23. https://doi.org/10.1016/j.jep.2013.05.048

198. Hua S, Zhang Y, Liu J, Dong L, Huang J, Lin D, Fu X. Ethnomedicine, Phytochemistry and Pharmacology of Smilax glabra: An Important Traditional Chinese Medicine. Am J Chin Med. 2018;46:261-97. https://doi.org/10.1142/S0192415X18500143

199. Biao CY, Ming ZD, Shan YS. Chemical Constituents of Smilax perfoliata. Acta Bot Sin. 2004;46:618-20.

200. Borkataky M. Antimcrobial and antioxidant activity of Smilax perfoliate Lour. Der Pharm Lett. 2014;6:246-50.

201. Zhao Y, Liu F, Lou HX. Studies on the chemical constituents of Solanum nigrum. Zhong Yao Cai. 2010;33:555-6.

202. Hameed HI, Calixto CMR, Yahya HM. A Review: Solanum nigrum L. Antimicrobial, Antioxidant properties, Hepatoprotective effects and Analysis of Bioactive Natural Compounds. Res J Pharm Tech. 2017;10:4063-8. http://dx.doi.org/10.5958/0974-360X.2017.00737.5

203. Jain RAS, Gupta SSPI, Gabrani R. Solanum nigrum: current perspectives on therapeutic properties. Altern Med Rev. 2011;16:78-85.

204. Chang FR, Yen CT, El-Shazly M, Yu CY, Yen MH, Cheng YB, Chen SL, Wu YC. Spirostanoids with 1,4-dien-3-one or 3ß,7a-diol-5,6-ene moieties from Solanum violaceum. Bioorg Med Chem Lett. 2013;23:2738-42. https://doi.org/10.1016/j.bmcl.2013.02.060

205. Karim A, Islam B, Tareq SM, Islam MT. Antinociceptive and antipyretic activities of Solanum violaceum Ortega. Int J Med. 2017;5:90-3. Doi:10.14419/ijm.v5i1.7282

206. Mahaldar K, SaifuzMahaldar K, Saifuzzaman M, Irin T, Barman AK, Islam MK, Rahman MM, Islam MA. Analgesic, anthelmintic and toxicity studies of Solanum violaceum Linn. leaves. Orient Pharm Exp Med. 2016;16:147-52. https://doi.org/10.1007/s13596-016-0227-9

207. Yen CT, Lee CL, Chang FR, Hwang TL, Yen HF, Chen CJ, Chen SL, Wu YC. Indiosides G-K: Steroidal Glycosides with Cytotoxic and Anti-inflammatory Activities from Solanum violaceum. J Nat Prod. 2012;75:636-43. https://doi.org/10.1021/np200877u

208. Bora NS, Kakoti BB, Gogoi B, Goswami AK. Ethno-medicinal claims, phytochemistry and pharmacology of Spondias pinnata: A review. Int J pharm Sci Res. 2014;5:1138-45. DOI: 10.13040/IJPSR.0975-8232.5(4).

209. Goswami DV, Nirmal SA, Patil MJ, Dighe NS, Laware NS, Patta SR. An Overview of Tectona grandis: Chemistry and Pharmacological Profile. Phcog Rev. 2009;3:181-5.

210. Vyas P, Yadav DK, Khandelwal P. Tectona grandis (Teak) - A review on its phytochemical and therapeutic potential. Nat Prod Res. 2019;33:2338-54.

211. El-Ghorab A, El-Massry KF, Shibamoto T. Chemical Composition of the Volatile Extract and Antioxidant Activities of the Volatile and Nonvolatile Extracts of Egyptian Corn Silk (Zea mays L.). J Agric Food Chem. 2007;55:9124-7. DOI: 10.1021/jf071646e

212. Mendoza-Díaz S, del Carmen Ortiz-Valerio M, Castaño-Tostado E, de Dios Figueroa-Cárdenas J, Reynoso-Camacho R, Ramos-Gómez M, Campos-Vega R, Loarca-Piña G. Antioxidant Capacity and Antimutagenic Activity of Anthocyanin and Carotenoid Extracts from Nixtamalized Pigmented Creole Maize Races (Zea mays L.). Plant Foods Hum Nutr. 2012;67:442-9. https://doi.org/10.1007/s11130-012-0326-9

213. Nessa F, Ismail Z, Mohamed N. Antimicrobial Activities of Extracts and Flavonoid Glycosides of Corn Silk (Zea mays L). Int J Biotechnol Wellness Ind. 2012;1:115-21.

214. Gupta SK, Sharma A. Medicinal properties of Zingiber officinale Roscoe - A Review. IOSR J Pharm Biol Sci. 2011;5:124-9.

215. Rehman R, Akram M, Akhtar N, Jabeen Q, Shah SA, Ahmed K, Shaheen G, Asif HM. Zingiber officinale Roscoe (Parmacological activity). J Med Plants Res. 2011;5:344-8.

216. Çakılcıoğlu U, Khatun S, Türkoğlu I, Hayta S. Ethnopharmacological survey of medicinal plants in Maden (Elazığ-Turkey). J Ethnopharmacol. 2011;137:469-86. https://doi.org/10.1016/j.jep.2011.05.046

217. Akindele AJ, Ibe IF, Adeyemi 0O. Analgesic and antipyretic activities of Drymaria cordata (Linn.) Willd (Caryophyllaceae) extract. Afr J Tradit Complement Altern Med. 2012;9:25-35.

218. Proma JJ, Faruque MO, Rahman S, Bashar ABMA, Rahmatullah M. Analgesic potential and phytochemical screening of Lablab purpureus aerial parts. World J Pharm Pharmaceut Sci. 2014;3:165-73. 
219. Bakir M, Facey PC, Hassan I, Mulder WH, Porter RB. Mikanolide from Jamaican Mikania micrantha. Acta Crystallographica Section C: Crystal Structure Communications. . 2004;6:11. DOI: 10.1107/S0108270104017809

220. Koriem KM, Arbid MS, Saleh HN. Antidiarrheal and protein conservative activities of Psidium guajava in diarrheal rats. J Integr Med. 2019;17:57-65. https://doi.org/10.1016/j.joim.2018.12.001

221. Salama AA, El-Kassaby MI, Hassan A. Anti-urolithiatic activity of Solanum nigrum hydroalcoholic extract in ethylene glycol-induced urolithiasis in rats. Egypt Pharmaceut J. 2019;18:311. https://www.epj.eg.net/text.asp?2019/18/4/311/272269.

222. Yirga G, Teferi M, Gidey G, Zerabruk S. An ethnoveterinary survey of medicinal plants used to treat livestock diseases in Seharti-Samre district, Northern Ethiopia. Afr J Plant Sci. 2012;6:113-9. https://doi.org/10.5897/AJPS11.242

223. Parthiban R, Vijayakumar S, Prabhu S, Gnanaselvam J, Yabesh EM. Quantitative traditional knowledge of medicinal plants used to treat livestock diseases from Kudavasal taluk of Thiruvarur district, Tamil Nadu, India. Rev Bras Farmacogn. 2016;26:109-21. https://doi.org/10.1016/j.bjp.2015.07.016

224. Jadid N, Kurniawan E, Himayani CES, Prasetyowati I, Purwani KI, Muslihatin W, Hidayati D, Tjahjaningrum ITD. An ethnobotanical study of medicinal plants used by the Tengger tribe in Ngadisari village, Indonesia. Plos one. 2020;15:7. https://doi.org/10.1371/journal.pone.0235886

225. Addo-Fordjour P, Kofi Anning A, Durosimi Belford EJ, Akonnor D. Diversity and conservation of medicinal plants in the Bomaa community of the Brong Ahafo region, Ghana. J Med Plants Res. 2008;2:226-33.

226. Ghorbani A. Studies on pharmaceutical ethnobotany in the region of Turkmen Sahra, north of Iran (Part 1): general results. J Ethnopharmacol. 2005;102:58-68. https://doi.org/10.1016/j.jep.2005.05.035

227. Poonam K, Singh GS. Ethnobotanical study of medicinal plants used by the Taungya community in Terai Arc Landscape, India. J Ethnopharmacol. 2009;123: 167-76. https://doi.org/10.1016/j.jep.2009.02.037

228. Luseba D, Tshisikhawe MP. Medicinal plants used in the treatment of live-stock diseases in Vhembe region, Limpopo province, S Afr J Med Plants Res. 2014;7: 593-601. https://doi.org/10.5897/JMPR12.1213

229. Mahwasane ST, Middleton L, Boaduo N. An ethnobotanical survey of indigenous knowledge on medicinal plants used by the traditional healers of the Lwamondo area, Limpopo province, South Africa. S Afr J Bot. 2013;88:69-75. https://doi.org/10.1016/j.sajb.2013.05.004

230. Saha MR, Sarker DD, Sen A. Ethnoveterinary practices among the tribal community of Malda district of West Bengal, India. Indian J Tradit Knowl. 2014;13: 359-67. http://nopr.niscair.res.in/handle/123456789/27931

231. Verma RK, Kumari P, Maurya RK, Kumar V, Verma RB, Singh RK. Medicinal properties of turmeric (Curcuma longa L.): A review. Int J Chem Stud. 2018;6(4):1354-7.

232. Singh AG, Kumar A, Tewari DD. An ethnobotanical survey of medicinal plants used in Terai forest of western Nepal. J Ethnobiol Ethnomedicine. 2012; 8:19. https://doi.org/10.1186/1746-4269-8-19

233. Chaudhary MI, He Q, Cheng YY, Xiao PG. Ethnobotany of medicinal plants from Tian Mu Shan biosphere reserve, Zhejiang-Province, China. Asian J Plant Sci. 2006;5:646-53.

234. Çakılcıoğlu U, Türkoğlu I. An ethnobotanical survey of medicinal plants in Sivrice (Elazığ-Turkey). J Ethnopharmacol. 2010;132:165-75. https://doi.org/10.1016/j.jep.2010.08.017

235. Rajakumar N, Shivanna MB. Ethnomedicinal application of plants in the eastern region of Shimoga District, Karnataka, India. J Ethnopharmacol. 2009;126:64-73. https://doi.org/10.1016/j.jep.2009.08.010

\section{Figures}




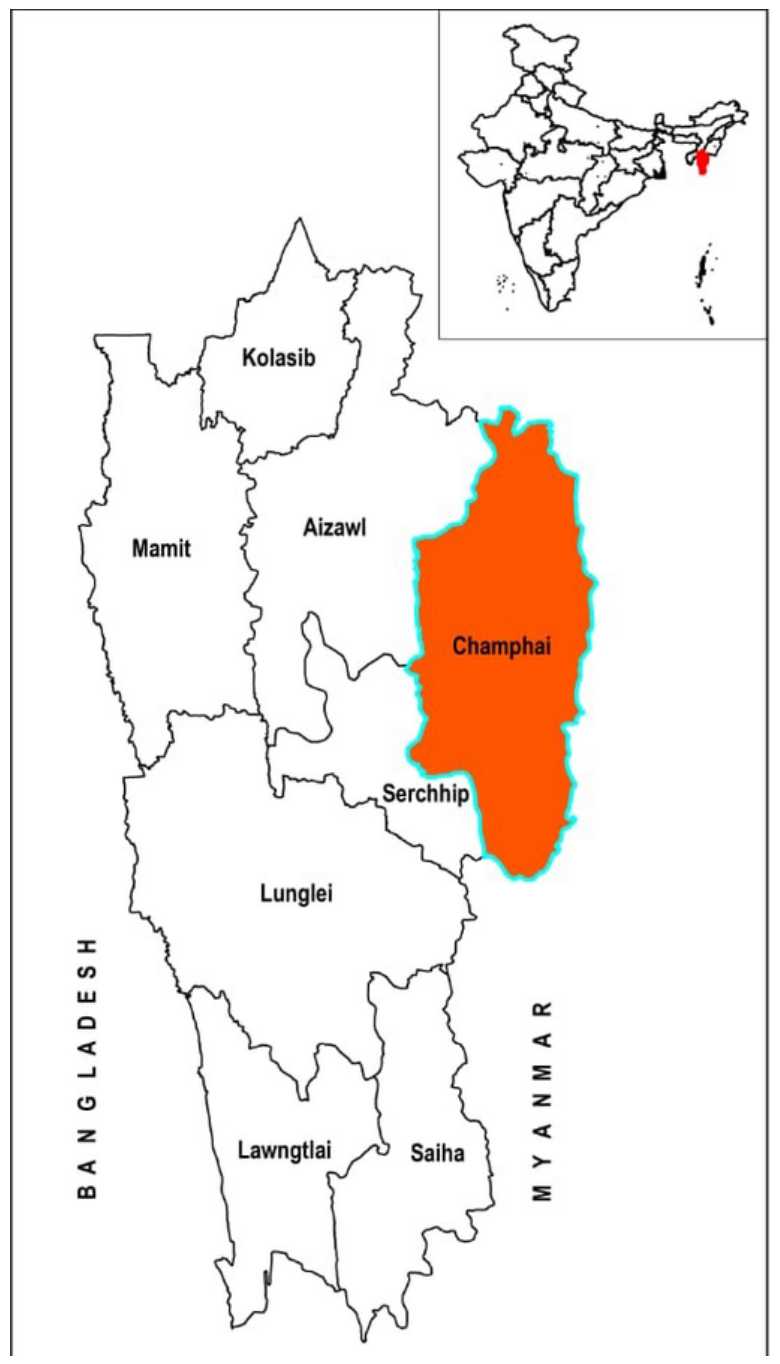

\section{Champhai District Sampling Site Map}

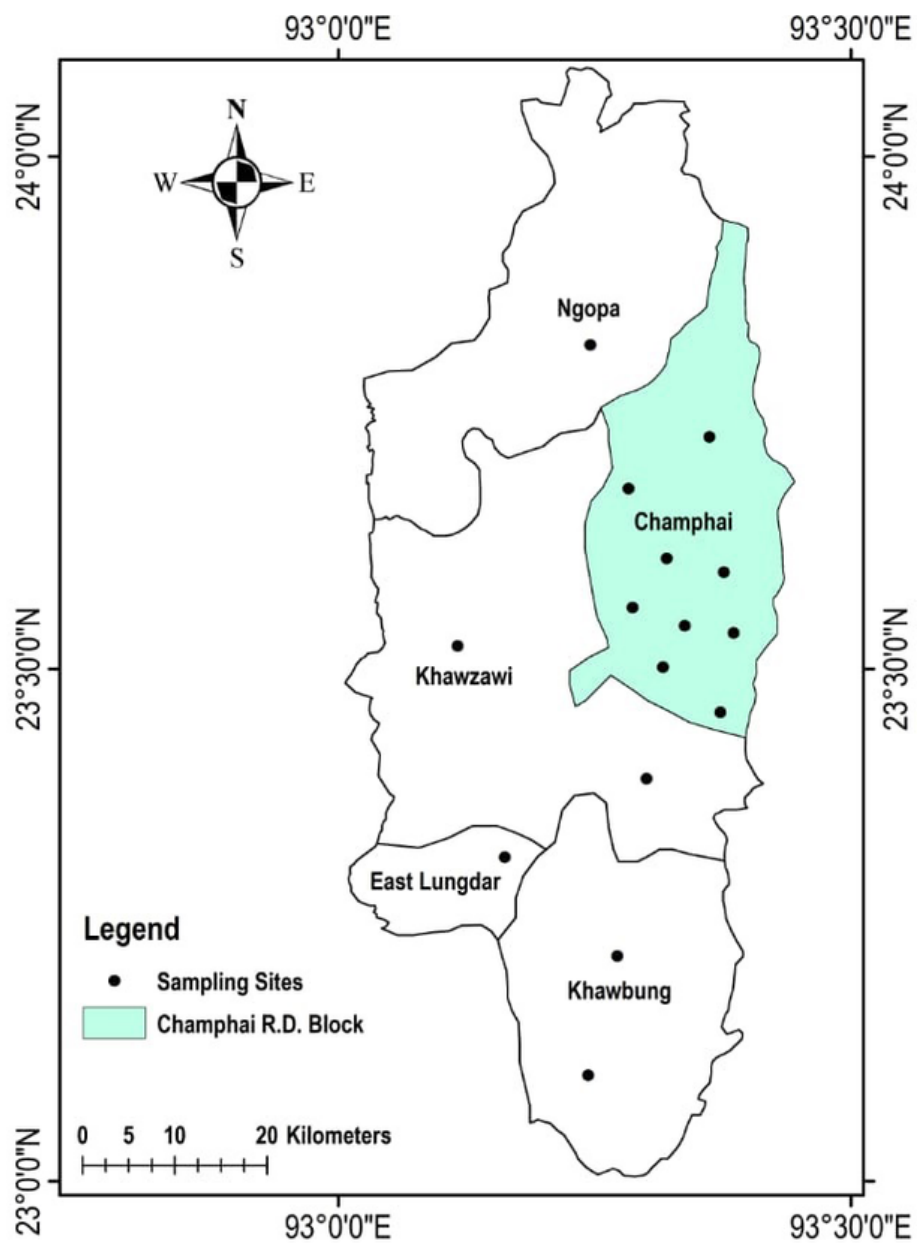

Figure 1

Location of the present study area: Champhai District, Mizoram, India

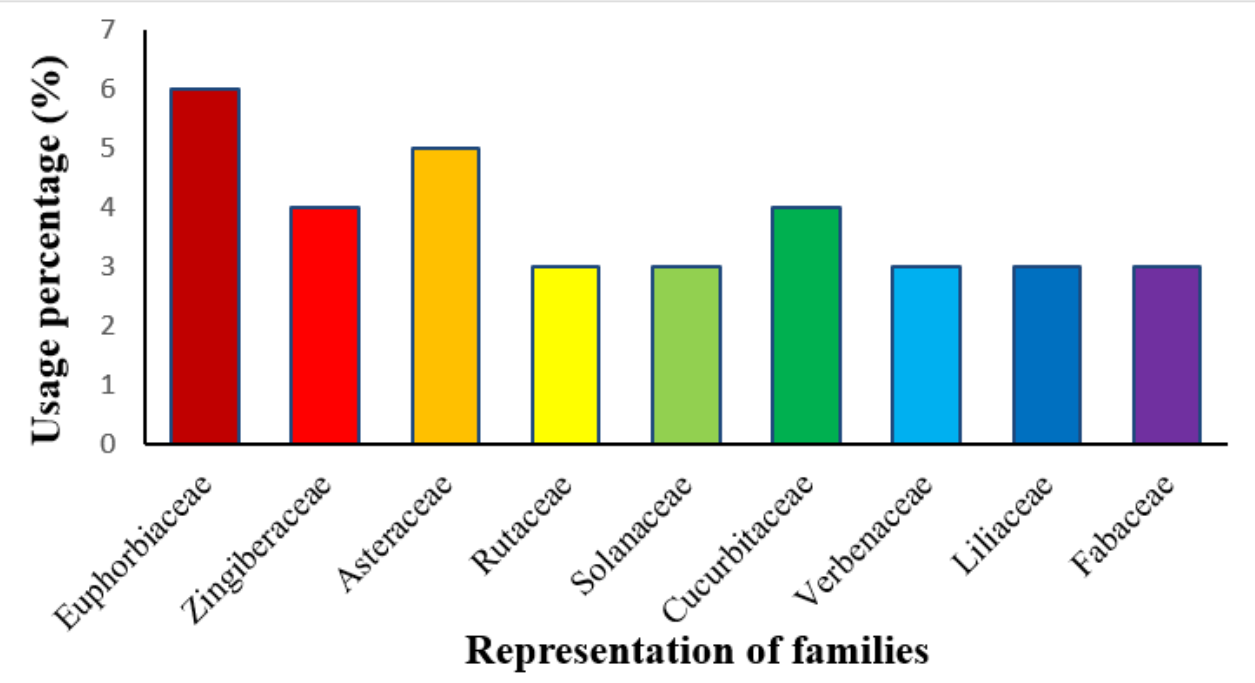

Figure 2

Distribution of plant usage based on the family 


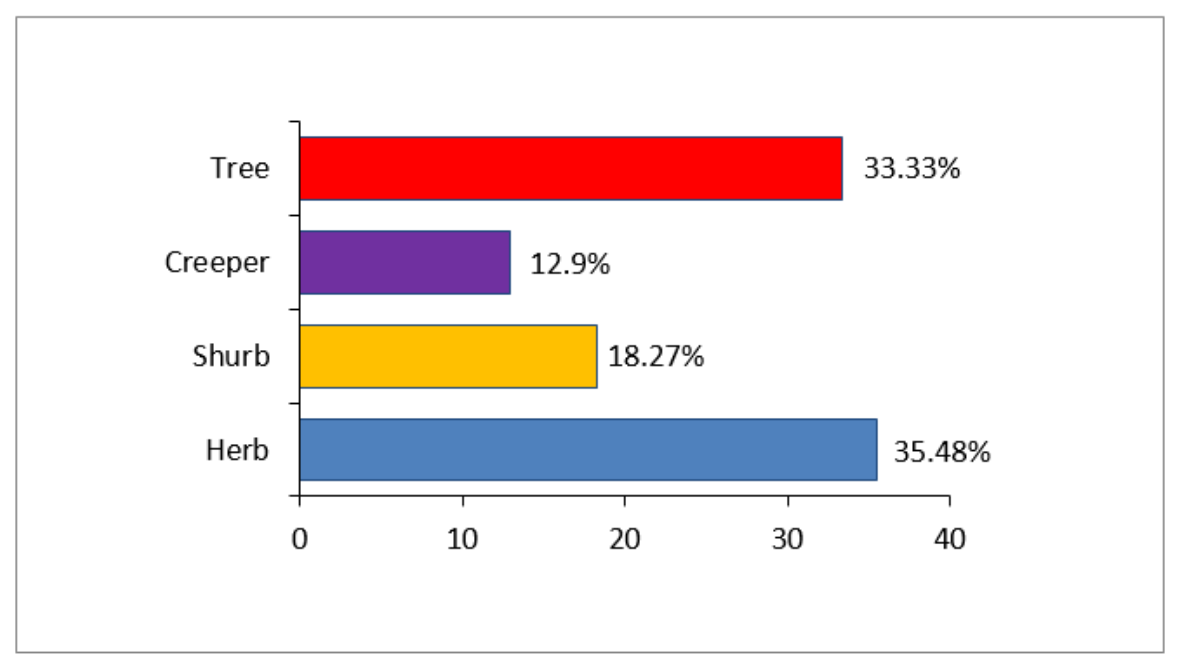

Figure 3

Percentage of plants habit.

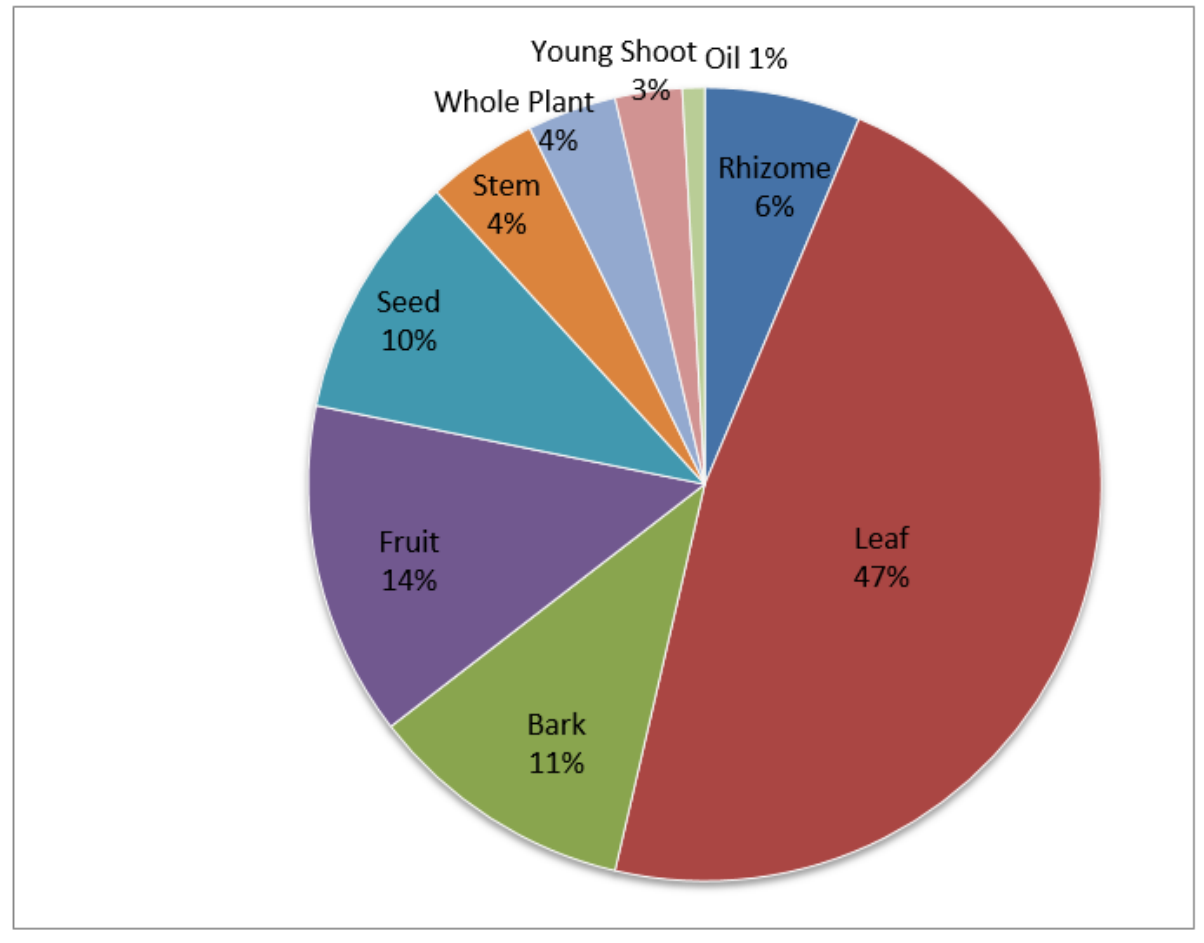

Figure 4

Percentage of parts used 


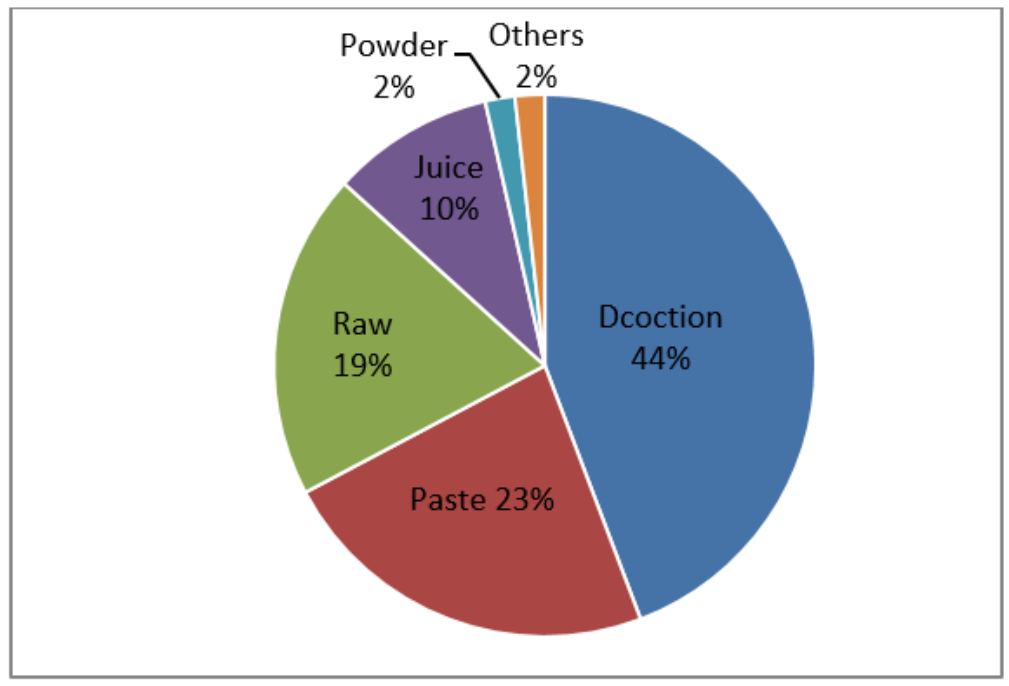

Figure 5

Distribution of formulation usage 